\title{
Meta-análise sobre Conhecimento para Ensinar Divisão de Frações
}

\section{Meta-analysis about knowledge to teaching fractions division}

\author{
Jeferson Gomes Moriel Junior* \\ ORCID iD 0000-0003-1526-8002 \\ Gladys Denise Wielewski* \\ ORCID iD 0000-0002-2473-2957 \\ José Carrillo*** \\ ORCID iD 0000-0001-7906-416X
}

\begin{abstract}
Resumo
O objetivo deste artigo é identificar as principais contribuições de estudos sobre conhecimento docente relativo ao ensino e à aprendizagem da divisão de frações e analisar como tais resultados contribuem para responder a pergunta: que conjunto de conhecimentos um professor precisa para ensinar e fazer aprender divisão de frações? Realizamos uma meta-análise qualitativa no referido tema (considerando tanto a dimensão matemática, quanto a didático-pedagógica) por meio de leituras sucessivas na íntegra e análise de conteúdo de um corpus de 58 estudos resultante da busca em seis bancos de dados (JSTOR, ERIC, Banco de Teses da CAPES, SciELO, Base de dados da Revista do Professor de Matemática e no Google Scholar). Os resultados obtidos fornecem a visão integradora de um conjunto de conhecimentos involucrados em cinco grandes blocos: 1. Definições e notações; 2. Algoritmos, suas justificações e outros procedimentos; 3. Interpretações e problemas associados; 4. Explicações instrucionais, propostas de ensino e recursos didáticos; 5. Aspectos da aprendizagem de estudantes. Concluímos explicitando a utilidade deste conjunto de conhecimentos especializados para ensinar divisão de frações para licenciandos, professores, formadores e pesquisadores, bem como sua importância para a melhoria da formação e para a valorização da profissão docente.
\end{abstract}

Palavras-chave: Meta-análise. Divisão de Frações. Ensino e Aprendizagem. Conhecimento Especializado de Professores.

\begin{abstract}
The aim of this article is to identify the main contributions of studies on teaching knowledge regarding the teaching and learning and to analyze how these results contribute to answering the question: which set of \footnotetext{
REAMEC). Professor do Instituto Federal de Mato Grosso (IFMT) e do Programa de Pós-graduação em Ensino do IFMT/UNIC, campus Cuiabá, Mato Grosso, Brasil. Endereço para correspondência: Rua Profa. Zulmira Canavarros, 95, Centro Norte, Cuiabá, MT, Brasil, CEP: 78048-076. E-mail: jeferson.moriel@cba.ifmt.edu.br.

** Doutora em Educação Matemática pela Pontifícia Universidade Católica de São Paulo (PUC). Professora do Depto. de Matemática e do Doutorado em Educação em Ciências e Matemática (REAMEC) da Universidade Federal de Mato Grosso (UFMT), Cuiabá, MT, Brasil. Endereço para correspondência: Av. Fernando Corrêa da Costa, 2367, Boa Esperança, Cuiabá, MT, Brasil, CEP: 78060-900. E-mail: gladysdw@ brturbo.com.br.

**** Doutor em Filosofia e Ciências da Educação pela Universidade de Sevilla (US). Catedrático de Didática da Matemática e do Programa de Doutorado em Investigação em Ensino e Aprendizagem das Ciências (IEACAD) da Universidade de Huelva (UHU), campus El Carmen, Huelva, Espanha. Endereço para correspondência: Avda. 3 de marzo, s/n, Huelva, España, E-21007. E-mail: carrillo@uhu.es.
}

* Doutor em Educação em Ciências e Matemática pela Universidade Federal de Mato Grosso (UFMT-
\end{abstract}


knowledge does a teacher need to teach and learn for fraction division? We performed a qualitative metaanalysis on this theme (considering both the mathematical and didactic-pedagogical dimension) through successive readings and content analysis of a corpus of 58 studies all resulting from the search in six databases (JSTOR, ERIC, CAPES Theses Bank, SciELO, Mathematics Teacher's Journal Database and Google Scholar). The results obtained provided the integrative vision of a set of knowledge involved in five large blocks, namely: 1. Definitions and notations; 2. Algorithms, their justifications and other procedures; 3. Interpretations and associated problems; 4. Instructional explanations, teaching proposals and didactic resources; 5. Aspects of student learning. We conclude the article by explaining the usefulness of this set of specialized knowledge to teach fraction division for graduates, teachers, trainers, and researchers, as well as their importance for improvement in the mathematic education and for the appreciation of the teaching profession.

Keywords: Meta-analysis. Fraction Division. Teaching and Learning. Teacher's Specialized Knowledge.

\section{Introdução}

A aprendizagem de frações é um processo longo e complexo, que não se restringe a um ou dois anos escolares, mas sim ocorre ao longo de todo o Ensino Fundamental (KILPATRICK; SWAFFORD; FINDELL, 2001; LOPES, 2008; MOREIRA; DAVID, 2005). Dificuldades e erros na aprendizagem dos números fracionários e suas operações são apontadas na literatura e em avaliações nacionais (BAYOUD, 2011; BERTONI, 2008; 2009; BRASIL, 2001; 2003; FÁVERO; PINA NEVES, 2012; NAISER; WRIGHT; CAPRARO, 2003; PINILLA, 2007; SANTOS; SANTOS; CAMPOS, 2013).

Ao longo dos últimos 20 anos, a avaliação nacional da Educação Básica (intitulada de Prova Brasil) tem mostrado que, em média, apenas $13 \%$ dos alunos terminaram o Ensino Fundamental, conseguindo resolver corretamente um problema com números racionais envolvendo suas operações (INEP, 2014a; 2014b) ${ }^{1}$. Das operações com frações, a divisão tem sido considerada por professores e alunos a mais mecânica e menos compreensível (ISIKSAL; CAKIROGLU, 2007; KRIBS-ZALETA, 2006; MA, 1999; OLANOFF, 2011; ÖZEL, 2013; PAYNE, 1976; TIROSH, 2000). Por vezes, ela é vista como um tópico com escassez de aplicações realísticas (LOPES, 2008), de difícil abordagem intuitiva (BALL, 1990) e o mais complexo da aritmética escolar (BULGAR, 2003; MA, 1999). Comumente professores e licenciandos em Matemática apresentam limitações de conhecimento relacionado ao tópico (que os levam, por exemplo, a cometer os mesmos erros de estudantes da Educação Básica) ou ao seu ensino (BALL, 1990; BAYOUD, 2011; MA, 1999).

Este cenário nos levou a questionar qual é o conjunto de conhecimentos que um professor deve ter para superar as limitações em questão e fazer com que os alunos aprendam divisão de frações. Recorremos à literatura na tentativa de compreender as contribuições que

\footnotetext{
${ }^{1}$ Média feita com todos os resultados do SAEB de 1995 a 2011 considerando os índices do nível 7 de proficiência que envolve conhecimento sobre operações com racionais.
} 
pesquisadores deram para responder tal questão, buscando obras de síntese como trabalhos de meta-análise, estados da arte ou pesquisas bibliográficas (LIMA; MIOTO, 2007) no campo de pesquisa relativo ao conhecimento docente sobre divisão de frações. Os dois estudos encontrados focalizam conteúdos correlatos, porém mais amplos do que o referido. Um deles revisou 160 pesquisas internacionais publicadas entre 1962 e 2004 sobre o tema frações (PINILLA, 2007), das quais apenas quatro eram específicas sobre divisão de frações, sem abordar especificamente conhecimento de professores. O outro explorou a temática divisão $e$ números racionais (FÁVERO; PINA NEVES, 2012), encontrando 65 estudos nacionais e internacionais publicados de 1999 a 2010, dos quais apenas um tinha como foco a divisão de frações, porém investigava alunos (SHARP; ADAMS, 2002).

Considerando que não encontramos alguma obra-síntese com o foco desejado, apresentamos neste artigo uma meta-análise sob o enfoque qualitativo (BICUDO, 2014) de estudos nacionais e internacionais centrados na temática conhecimento docente sobre divisão de frações. O objetivo deste trabalho é identificar as principais contribuições de estudos sobre conhecimento docente relativo ao ensino e a aprendizagem da divisão de frações e analisar como tais resultados contribuem para responder a pergunta: que conjunto de conhecimentos um professor precisa para ensinar e fazer aprender divisão de frações? A presente investigação é um aprofundamento de uma pesquisa que buscou caracterizar o conhecimento especializado para ensinar divisão de frações contemplando também dados empíricos de professores e futuros professores de Matemática (MORIEL JUNIOR, 2014).

\section{Encaminhamento metodológico}

Utilizamos a meta-análise qualitativa como procedimento metodológico que consiste na revisão sistemática de um conjunto de pesquisas sobre um dado tema, cuja finalidade é integrar os resultados desses estudos e produzir sínteses mediante o confronto dos mesmos, possibilitando assim maior visibilidade, impacto da produção e ampliação da generalização dos resultados existentes (BICUDO, 2014; FIORENTINI; LORENZATO, 2006).

A busca por trabalhos foi realizada em seis bancos de dados (JSTOR, ERIC, Banco de Teses da CAPES, Scielo, Base de dados da Revista do Professor de Matemática ${ }^{2}$ e no Google Scholar $^{3}$ ) durante o mês de outubro de 2014. Considerando nosso tema de interesse (conhecimento docente sobre divisão de frações) e partindo do pressuposto teóricoepistemológico de que o conhecimento do professor de Matemática é caracterizado tanto pela

\footnotetext{
${ }^{2}$ Banco de dados digital com as 70 primeiras edições publicadas no periódico, de 1982 a 2009 (RPM, 2011).

${ }^{3}$ Ferramenta de indexação de publicações científicas (http://scholar.google.com).
} 
dimensão do conhecimento do conteúdo, quanto do didático-pedagógico (CARRILLO et al., 2014; SHULMAN, 1986), optamos por utilizar nas buscas as seguintes palavras-chave: divisão, division, divisão de frações, fraction division, division of fraction, division with fraction, división de fracciones, conhecimento, knowledge, conocimiento, número racional, rational, divisão de racionais, operações e operations (sozinhas ou em combinação). A partir de então, leituras sucessivas de todo material encontrado (incluindo aquelas de reconhecimento, exploratória, seletiva e interpretativa) foram feitas para obter as informações necessárias e verificar a relevância e pertinência de cada trabalho em relação ao propósito da pesquisa (BICUDO, 2014; FIORENTINI; LORENZATO, 2006; SALVADOR, 1986).

Este encaminhamento possibilitou constituir um corpus de 58 estudos na íntegra ${ }^{4}$ incluindo teses, dissertações, livros, artigos publicados em revistas de professores, periódicos científicos e anais de eventos. Os trabalhos foram classificados em dois grupos conforme seu foco (Tabela 1). O primeiro abrange aqueles que investigam o conhecimento docente sobre divisão de frações cujos sujeitos da pesquisa são professores, licenciandos ou formadores para o ensino de Matemática. O segundo grupo engloba trabalhos cujo foco não está nos referidos sujeitos, mas abordam aspectos matemáticos, do ensino ou da aprendizagem relacionados à divisão de fração, como algoritmos, suas justificativas e problemas associados à operação, abordagens instrucionais, análise de recursos didáticos em si, erros comuns de alunos etc.

Tabela 1 - Relação de trabalhos revisados classificados por temática.

\begin{tabular}{|c|c|c|}
\hline FOCO & & QUANTIDADE (REFERÊNCIAS) \\
\hline $\begin{array}{c}\text { Conhecimento } \\
\text { docente sobre divisão } \\
\text { de frações }\end{array}$ & 29 & $\begin{array}{l}\text { (BALL, 1990; BARBOSA, 2011; BAYOUD, 2011; CHARALAMBOUS; HILL; } \\
\text { BALL, 2011; CHINNAPPAN; DESPLAT, 2012; GARCÍA, 2013; GREEN; } \\
\text { PIEL; FLOWERS, 2008; ISIKSAL; CAKIROGLU, 2007; KRIBS-ZALETA, } \\
\text { 2006; LI; KULM, 2008; LIN et al., 2013; LO; LUO, 2012; LO; MCCRORY, } \\
\text { 2010; LUBINSKI; FOX; THOMASON, 1998; LUO; LO; LEU, 2011; MA, 1999; } \\
\text { NEWTON, 2008; NILLAS, 2003; OLANOFF, 2011; ÖZEL, 2013; PIEL; } \\
\text { GREEN, 1994; REDMOND, 2009; RIZVI; LAWSON, 2007; RULE; } \\
\text { HALLAGAN, 2006; SALINAS, 2009; SHARON; SWARTHOUT, 2014; } \\
\text { SILVEIRA; SILVA, 2013; TCHOSHANOV, 2011; TIROSH, 2000) }\end{array}$ \\
\hline $\begin{array}{l}\text { Aspectos da divisão } \\
\text { de frações, seu ensino } \\
\text { e aprendizagem }\end{array}$ & 29 & $\begin{array}{l}\text { (AKSU, 1997; ARAÚJO, 2013; ASHLOCK, 2006; BERTONI, 2009; BULGAR, } \\
\text { 2003; CHEN et al., 2013; CONTRERAS, 2012; FLORES, 2008; FLORES, 2013; } \\
\text { GUERRA; SILVA, 2008; HIRATSUKA, 1996; KILPATRICK; SWAFFORD; } \\
\text { FINDELL, 2001; LI, 2008; LI; CHEN; AN, 2009; LI; CHEN; KULM, 2009; } \\
\text { LIMA, 1983; LOPES, 2008; MADEIRO, 1996; MOREIRA, 2013; MOREIRA; } \\
\text { FERREIRA, 2008; NAISER; WRIGHT; CAPRARO, 2003; PAYNE, 1976; } \\
\text { PERÉZ, 2009; PHILIPP, 2000; PURITZ, 2005; SÁ, 2015; SHARP; ADAMS, } \\
\text { 2002; SILVA; ALMOULOUD, 2008; SINICROPE; MICK; KOLB, 2002) }\end{array}$ \\
\hline TOTAL & 58 & - \\
\hline
\end{tabular}

\footnotetext{
${ }^{4}$ Quatro trabalhos encontrados (BALL, 1988; CONTRERAS, 2004; CONTRERAS; ALFONSO, 2005; LI; SMITH, 2007) não foram integrados ao estudo por tratar-se de um tipo de versão prévia de outros mais atuais e completos que efetivamente integram nosso corpus. Um trabalho (KAMII; WARRINGTON, 1995) não foi encontrado na íntegra, por isso ele não faz parte do corpus.
} 
Considerando que nosso foco é o conhecimento docente, é importante caracterizar as tendências do primeiro grupo da tabela anterior. Iniciamos identificando escassez de estudos nacionais (BARBOSA, 2011; SILVEIRA; SILVA, 2013). Em relação aos objetivos principais das pesquisas, podem ser agrupadas nas seguintes linhas, em ordem decrescente de frequência (Tabela 3): 1. caracterizar o conhecimento de licenciandos, professores e formadores (envolvendo exploração, identificação e descrição); 2. avaliar a efetividade de uma formação oferecida (envolvendo disciplinas de graduação ou cursos rápidos); 3. Comparar o conhecimento de sujeitos de diferentes nacionalidades ou etapas da profissão (graduandos e graduados).

A perspectiva teórica sobre o conhecimento de professores (Tabela 2) mais presente é a de Shulman (1986), que é genérica e não diz respeito a uma área específica, seguida pelo modelo Mathematical Knowledge for Teaching - MKT (BALL; HILL; BASS, 2005; BALL; THAMES; PHELPS, 2008), que é específico para a Matemática e fundado na perspectiva anterior. Esses dois marcos teóricos alicerçam a maioria (77\%) dos estudos que visam caracterizar o conhecimento docente (Tabela 4). Em termos de conteúdo, mais da metade das produções aborda exclusivamente a divisão de frações, enquanto o restante discute também outras operações ou conceitos (Tabela 5). Predomina a exploração do domínio matemático em detrimento do didático (Tabela 5). Por fim, computamos que o corpus investigado contempla conhecimento de 999 licenciandos, 390 professores e 4 formadores, totalizando 1393 sujeitos.

Tabela 2 - Perspectivas teóricas sobre conhecimento docente que fundamentam os trabalhos

\begin{tabular}{ccc}
\hline PERSPECTIVA TEÓRICA & QTDE. & TRABALHOS \\
Shulman (1986) & $12(41 \%)$ & $\begin{array}{c}\text { (BALL, 1990; ISIKSAL; CAKIROGLU, 2007; LIN et al., 2013; } \\
\text { MA, 1999; ÖZEL, 2013; REDMOND, 2009; RIZVI; LAWSON, } \\
\text { 2007; SILVIRA; SILVA, 2013; TCHOSHANOV, 2011; TIROSH, } \\
\text { 2000) (NILLAS, 2003; SHARON; SWARTHOUT, 2014)* }\end{array}$ \\
\hline MKT & $10(35 \%)$ & $\begin{array}{c}\text { CHAYOUD, 2011; CHARALAMBOUS; HILL; BALL, 2011; } \\
\text { CHINNAPAN; DESPLAT, 2012; GARCIA, 2013; LI; KULM, } \\
\text { 2008; LO; LUO, 2012; LUO; LO; LEU, 2011; NEWTON, 2008; } \\
\text { OLANOFF, 2011; SALINAS, 2009) }\end{array}$ \\
\hline Não esclarecido & $6(21 \%)$ & $\begin{array}{c}\text { (GREEN; PIEL; FLOWERS, 2008; KRIBS-ZALETA, 2006; LO; } \\
\text { MCCRORY, 2010; LUBINSKI; FOX; THOMASON, 1998; PIEL; } \\
\text { GREEN, 1994; RULE; HALLAGAN, 2006) }\end{array}$ \\
\hline Outro: MTCS & $1(3 \%)$ & (BARBOSA, 2011) \\
\hline TOTAL & $29(100 \%)$ & - \\
\hline Fonte: Dados organizados pelo autor a partir do corpus de estudos revisado (2019). \\
Observação: *Dois trabalhos que usam Ma (1999), cuja base é Shulman (1986)
\end{tabular}

Tabela 3 - Objetivos principais das investigações sobre conhecimento docente relativo à divisão de frações

\begin{tabular}{ccc}
\hline $\begin{array}{c}\text { OBJETIVOS DE } \\
\text { ESTUDO }\end{array}$ & QTDE. & \multicolumn{1}{c}{ TRABALHOS } \\
\hline $\begin{array}{c}\text { Caracterização do } \\
\text { conhecimento }\end{array}$ & $18(62 \%)$ & (BALL, 1990; BARBOSA, 2011; CHARALAMBOUS; HILL; BALL, \\
& & 2011; CHINNAPPAN; DESPLAT, 2012; GARCÍA, 2013; ISIKSAL; \\
& CAKIROGLU, 2007; KRIBS-ZALETA, 2006; LI; KULM, 2008; LO; \\
LUO, 2012; LUBINSKI; FOX; THOMASON, 1998; NEWTON, 2008; \\
\hline
\end{tabular}




\begin{tabular}{|c|c|c|}
\hline & & $\begin{array}{l}\text { NILLAS, 2003; OLANOFF, 2011; ÖZEL, 2013; PIEL; GREEN, 1994; } \\
\text { SALINAS, 2009; SILVEIRA; SILVA, 2013; TCHOSHANOV, 2011) }\end{array}$ \\
\hline $\begin{array}{l}\text { Efetividade da } \\
\text { formação }\end{array}$ & $7(24 \%)$ & $\begin{array}{c}\text { (GREEN; PIEL; FLOWERS, 2008; LO; MCCRORY, 2010; REDMOND, } \\
\text { 2009; RIZVI; LAWSON, 2007; RULE; HALLAGAN, 2006; SHARON; } \\
\text { SWARTHOUT, 2014; TIROSH, 2000) }\end{array}$ \\
\hline $\begin{array}{l}\text { Comparação entre } \\
\text { sujeitos }\end{array}$ & $4(14 \%)$ & (BAYOUD, 2011; LIN et al., 2013; LUO; LO; LEU, 2011; MA, 1999) \\
\hline TOTAL & $29(100 \%)$ & - \\
\hline
\end{tabular}

Tabela 4 - Descrição dos tipos de estudo por perspectiva teórica de conhecimento docente

\begin{tabular}{cccccc}
\hline TiPO DE ESTUDO & SHUlman & MKT & $\begin{array}{c}\text { NÃo } \\
\text { ESCLARECIDo }\end{array}$ & OUTRO & TOTAL \\
\hline Caracterização do conhecimento & $6(33 \%)$ & $8(44 \%)$ & $3(17 \%)$ & $1(6 \%)$ & $18(100 \%)$ \\
\hline Efetividade da formação & $4(57 \%)^{*}$ & - & $3(43 \%)$ & - & $7(100 \%)$ \\
\hline Comparação entre sujeitos & $2(50 \%)^{*}$ & $2(50 \%)$ & - & - & $4(100 \%)$ \\
\hline \multicolumn{6}{c}{ Fonte: Dados organizados pelo autor a partir do corpus de estudos revisado (2019). } \\
Observação: * Metade usa Ma (1999), cuja base é Shulman (1986)
\end{tabular}

Tabela 5 - Descrição dos domínios de conhecimento investigados por conteúdo matemático

\begin{tabular}{cccc}
\hline CONTEÚDO ABORDADO & $\begin{array}{c}\text { CONHECIMENTO } \\
\text { MATEMÁTICO }\end{array}$ & $\begin{array}{c}\text { CONHECIMENTO } \\
\text { MATEMÁTICO E } \\
\text { DIDÁTICO }\end{array}$ & TOTAL \\
\hline Somente divisão de frações & $9(31 \%)$ & $7(24 \%)$ & $16(55 \%)$ \\
\hline Divisão de frações e outros conteúdos & $7(24 \%)$ & $6(21 \%)$ & $13(45 \%)$ \\
\hline ToTAL & $16(55 \%)$ & $13(45 \%)$ & $29(100 \%)$ \\
\hline
\end{tabular}

Fonte: Dados organizados pelo autor a partir do corpus de estudos revisado (2019).

Descrevemos os resultados na seção seguinte contemplando todo o corpus em questão, esclarecendo que um mesmo trabalho pode figurar em mais de um bloco temático, diante da multiplicidade de contribuições que ele oferece.

\section{Resultados}

Após o processo de leituras sucessivas, análise do conteúdo e inventário do corpus, identificamos a emergência de cinco grandes blocos temáticos referentes às principais contribuições que os trabalhos oferecem, nomeadamente, no âmbito de: 1. Definições e notações; 2. Algoritmos, suas justificações e outros procedimentos; 3. Interpretações e problemas associados; 4. Explicações instrucionais, propostas de ensino e recursos didáticos; 5. Aspectos da aprendizagem de estudantes. Discutimos tais blocos temáticos a seguir.

\subsection{Definições e notações}


Embora não saibamos a origem exata, a divisão de frações está presente na China e no antigo Egito desde a antiguidade, associada à resolução de problemas, e tornou-se um objeto matemático cuja definição formal pode ser construída a partir do conjunto dos números inteiros usando classes de equivalências. Este tipo de conexão entre Matemática elementar e avançada faz parte do conhecimento matemático do professor de Matemática e é fundamental para o desenvolvimento da compreensão profunda da matemática fundamental (MA, 1999). Em síntese, trata-se da consciência da estrutura conceitual inerente a um conteúdo de Matemática elementar e a habilidade de fornecer um fundamento para tal estrutura conceitual.

No caso da divisão de frações, a estrutura conceitual subjacente e seus fundamentos foram identificados no corpus e estão sintetizados a seguir (CONTRERAS, 2012; GARCÍA, 2013). Estabelecida a relação de equivalência ${ }^{5}(a, b) R(c, d) \leftrightarrow a \cdot d=b \cdot c$ no conjunto $\mathbb{Z} \times$ $\mathbb{Z}^{*}=\left\{(a, b) / a \in \mathbb{Z}, b \in \mathbb{Z}^{*}\right\}$, temos como consequência as classes de equivalência por ela gerada em $\mathbb{Z} \times \mathbb{Z}^{*}$ e o conjunto quociente ${ }^{6} \mathbb{Z} \times \mathbb{Z}^{*} / R$, o qual passa a se denominar conjunto dos números racionais e denotar por $\mathbb{Q}$. Uma classe de equivalência de $\mathbb{Q}$ é chamada de número racional e denotada por $\overline{(a, b)}$, de modo que $\frac{a}{b}$ seja um representante desta classe de equivalência e chamado fração. O conjunto quociente $\mathbb{Z} \times \mathbb{Z}^{*} / R$ (dos números racionais, $\mathbb{Q}$ ) é um corpo, pois satisfaz as propriedades para tal (comutativa, associativa, elemento neutro, elemento inverso e distributiva) e porque podemos definir a operação de adição como $\overline{(a, b)}+\overline{(c, d)}=\overline{(a d+b c, b d)}$ e a de multiplicação como $\overline{(a, b)} \cdot \overline{(c, d)}=\overline{(a c, b d)}$. A existência da operação multiplicação e do inverso multiplicativo permite definir a divisão de frações como a multiplicação do dividendo pelo inverso do divisor, da seguinte forma: $\overline{(a, b)} \div \overline{(c, d)}=\overline{(a, b)} \cdot \overline{(c, d)}^{-1}=\overline{(a, b)} \cdot \overline{(d, c)}=\overline{(a d, b c)}$. Usando a notação de frações, isto pode ser reescrito como: $\frac{a}{b} \div \frac{c}{d}=\frac{a}{b} \cdot \frac{d}{c}=\frac{a d}{b c}$.

Outro modo de definir a divisão de frações, destacado em Moreira e Ferreira (2008), seria usando o subconstructo ${ }^{7}$ operador, a composição de funções lineares e a multiplicação de racionais. Embora não seja construída tal definição, ele exemplifica que para dividir $\frac{2}{3}$ por $\frac{7}{8}$ deve-se encontrar um operador $\mathrm{K}$ que leve $\frac{7}{8} \mathrm{em} \frac{2}{3}$. Isto seria obtido da seguinte forma: “o operador $\frac{2}{3}$ leva o 1 em $\frac{2}{3}$; o operador $\frac{8}{7}$ leva $\frac{7}{8}$ em 1 ; então, compondo, vemos que o operador

\footnotetext{
${ }^{5}$ Por ser reflexiva, simétrica e transitiva.

${ }^{6}$ É o conjunto de todas as classes de equivalência em $\mathbb{Z} \times \mathbb{Z}^{*}$ segundo $\mathrm{R}$.

7 Este termo diz respeito a uma das possíveis interpretações de frações que, embora tenham variado em quantidade e nomenclatura ao longo dos anos, são atualmente cinco as principais: relação parte-todo, medida, razão, quociente indicado e operador (MOREIRA; FERREIRA, 2008).
} 
$\frac{2}{3} \times \frac{8}{7}$ levará $\frac{7}{8}$ em $\frac{2}{3}$. Assim, $\frac{2}{3}$ dividido por $\frac{7}{8}$ é $\frac{2}{3} \times \frac{8}{7}$ " (MOREIRA; FERREIRA, 2008, p. 112). Generalizando essa ideia, temos que $\frac{\mathrm{p}}{\mathrm{q}}: \frac{\mathrm{r}}{\mathrm{s}}$ corresponde ao operador $\mathrm{K}$ que satisfaz $\mathrm{K} \cdot \frac{\mathrm{r}}{\mathrm{s}}=\frac{\mathrm{p}}{\mathrm{q}}$, $\mathrm{o}$ que implica em termos $K=\frac{\text { p.s }}{q . r}$, visto que $\frac{\text { p.s }}{q \cdot r} \cdot \frac{r}{s}=\frac{\text { p.s.r }}{\text { q.r.s }}=\frac{p}{q}$.

Nenhuma pesquisa do corpus investigou o conhecimento de (futuros) docentes sobre o conceito de divisão de frações neste nível avançado de formalização. Tampouco, foi abordado o fato de ela não ser considerada axiomaticamente uma operação nos racionais ${ }^{8}$, mas ser tratada como tal na Educação Básica. O que os estudos detectam é que o conhecimento conceitual dos sujeitos é limitado e, frequentemente, se resume ao modo memorizado de fazer o cálculo (BALL, 1990; MA, 1999; REDMOND, 2009; SILVEIRA; SILVA, 2013).

A maioria sabe calcular, mas não sabe explicar o significado da divisão de frações (BALL, 1990; GREEN; PIEL; FLOWERS, 2008; LI; KULM, 2008; LIN et al., 2013; LUO; LO; LEU, 2011; NILLAS, 2003; TIROSH, 2000). Isto é um dos indicadores de que geralmente o conhecimento é mais procedimental do que conceitual (GARCÍA, 2013; ÖZEL, 2013; RULE; HALLAGAN, 2006; SHARON; SWARTHOUT, 2014; TCHOSHANOV, 2011). Ainda assim, licenciandos e professores cometeram diversos erros procedimentais ao calcular divisão de frações. Os principais encontrados em Newton (2008), em ordem decrescente de frequência, são: (i) manter denominadores quando são iguais (por exemplo, $\frac{9}{10} \div \frac{3}{10}=\frac{3}{10}$ ); (ii) inverter o dividendo ao invés do divisor (inclusive quando era um inteiro, como em $4 \div \frac{1}{4}$ ); (iii) escrever $4 \div \frac{1}{4}=1$ sem explicar o motivo; (iv) inverter corretamente e multiplicar em cruz; (v) cancelar ou dividir em cruz (como em $\frac{2}{9} \div \frac{3}{8}=\frac{3}{4}$ ). Redmond (2009) também detectou o segundo deles, além de um procedimento equivocado de multiplicação cruzada. Em Salinas (2009), um sujeito inverteu a primeira, multiplicou pela segunda e ao final, inverteu a solução $\left(\frac{3}{4} \div \frac{1}{2}=\frac{3}{4} \cdot \frac{2}{1}=\frac{6}{4}=\frac{4}{6}\right)$.

Melhoras significativas no conhecimento procedimental/conceitual dos sujeitos sobre divisão de frações foram obtidas por meio de contextos formativos com atividades fundadas em resultados de pesquisas na área e incluíam trabalho com materiais manipulativos,

\footnotetext{
${ }^{8}$ A divisão de fraç̃oes é um caso particular de uma das operações (a multiplicação) que conferem ao conjunto dos números racionais a estrutura de corpo. Para ser uma operação em um conjunto E não vazio é preciso ser uma aplicação do tipo $f: E \times E \rightarrow E$. Logo, a adição e a multiplicação são operações em $\mathbb{Q}(f: \mathbb{Q} \times \mathbb{Q} \rightarrow \mathbb{Q}$ tal que $f(x, y)=x+y$ ou $x \cdot y)$, mas a divisão não, pois é caracterizada como $f: \mathbb{Q} \times \mathbb{Q}^{*} \rightarrow \mathbb{Q}$ tal que $f(x, y)=$ $\frac{x}{y}$. Assim, a divisão de frações só pode ser considerada uma operação em $\mathbb{Q}^{*}$, ou seja, se for definida como $f: \mathbb{Q}^{*} \times \mathbb{Q}^{*} \rightarrow \mathbb{Q}^{*}$ tal que $f(x, y)=\frac{x}{y}$. A implicação prática disto é que a operação de divisão de fraçães não pode fornecer a resposta para o cálculo envolvendo zeros, como $0: 1 / 2$, ou resultar zero, já que isto não está definido pela aplicação. Todavia, enquanto objeto de ensino na Educação Básica, a divisão é considerada uma operação com restrição de que o denominador não seja nulo.
} 
problemas e representações (LUBINSKI; FOX; THOMASON, 1998; NEWTON, 2008; REDMOND, 2009; RIZVI; LAWSON, 2007; RULE; HALLAGAN, 2006; SHARON; SWARTHOUT, 2014), reflexão sobre o porquê da divisão de frações (BARBOSA, 2011; TIROSH, 2000) podendo, inclusive, ter curta duração (GREEN; PIEL; FLOWERS, 2008).

Em termos de notação, encontramos a típica que utiliza dois pontos, com ou sem traço no meio, (como em $\frac{3}{4} \div \frac{1}{2}$ ), a fração complexa de modo que o dividendo seja separado por um traço do divisor (como em $\frac{\frac{1}{2}}{\frac{3}{4}}$ ) e apenas dois casos em que foi utilizada uma chave para separar dividendo e divisor, como em $6 \mid \underline{\frac{2}{3}}$ (MADEIRO, 1996) e em $\left.\frac{5}{5}\right|_{\underline{2}} ^{\frac{2}{5}}$ (FLORES, 2013).

\subsection{Algoritmos, suas justificações e outros procedimentos}

Em relação aos algoritmos da divisão de frações (cf. Tabela 6), um amplo inventário feito na literatura (CONTRERAS, 2012) identificou seis principais: 1. inversão da multiplicação que chamaremos de inverter e multiplicar (IM); 2. redução das frações ao um denominador comum e divisão dos numeradores que chamaremos de igualar denominadores (ID); 3. conversão das frações em decimais (CD) cuja diferença para o anterior é que o denominador comum é uma potência de 10; 4. produtos cruzados (PC); 5. uso da unidade fracionada (UF) para descobrir o valor unitário ${ }^{9}$ de uma grandeza em função de outra; 6. uso do protocolo da calculadora científica (CALC). Um sétimo algoritmo - dividir numeradores e denominadores entre si (DND) - é abordado em outros trabalhos (FLORES, 2008; GARCÍA, 2013; LI; KULM, 2008; ÖZEL, 2013; SILVA; ALMOULOUD, 2008; TIROSH, 2000).

Tabela 6 - Sete algoritmos da divisão de frações identificados

\begin{tabular}{|c|c|}
\hline NOMENCLATURA (SIGLA) & ALGORITMO \\
\hline Inverter e multiplicar (IM) & $\frac{a}{b} \div \frac{c}{d}=\frac{a}{b} \cdot \frac{d}{c}=\frac{a \cdot d}{b \cdot c}$ \\
\hline Igualar denominadores (ID) & $\frac{a}{b} \div \frac{c}{d}=\frac{a . d}{b . d} \div \frac{c . b}{d . b}=\frac{a . d}{b . c}$ \\
\hline $\begin{array}{c}\text { Conversão das frações em } \\
\text { decimais (CD) }\end{array}$ & $\frac{a}{b} \div \frac{c}{d}=\frac{a \cdot x}{b \cdot x} \div \frac{c \cdot y}{d \cdot y}=\frac{a x}{c y}=\frac{a \cdot d}{b \cdot c}$, sendo $b . x=d . y$ potências de 10. \\
\hline Produtos cruzados (PC) & $\frac{a}{b} \div \frac{c}{d}=\frac{a}{b} \times \frac{c}{d}=\frac{a . d}{b \cdot c}$ \\
\hline $\begin{array}{l}\text { Uso da unidade fracionada } \\
\text { (UF) }\end{array}$ & Se $\frac{a}{b}$ equivale a $\frac{c}{d}$, então $\frac{1}{d}$ equivale a $\frac{a}{b . c}$ e a unidade $\frac{d}{d}$ equivale a $\frac{a . d}{b . c}$ \\
\hline $\begin{array}{l}\text { Uso do protocolo da } \\
\text { calculadora científica (CALC) }\end{array}$ & $2 a \% 3 \div 1 a \% 5=10\lrcorner 3$. \\
\hline
\end{tabular}

\footnotetext{
${ }^{9}$ Termos empregado no sentido de taxa (razão que compara duas quantidades de magnitudes diferentes) em que o denominador é 1 . Como exemplos, $20 \mathrm{~km} / \mathrm{h}$ e 20 reais $/ \mathrm{kg}$.
} 


\section{Dividir numeradores e}

denominadores entre si (DND) $\frac{a}{b} \div \frac{c}{d}=\frac{a \div c}{b \div d} \quad($ Caso particular: $a$ é múltiplo de $c$ e $b$ é de $d)$
$\frac{a}{b} \div \frac{c}{d}=\frac{a . c . d}{b . c . d} \div \frac{c}{d}=\frac{a c d \div c}{b c d \div d}$

Fonte: Dados organizados pelo autor a partir do corpus de estudos revisado (2019).

É importante destacar que existem três regras mnemônicas associadas ao algoritmo inverte-e-multiplica: multiplicar em cruz $\left(\frac{a}{b} \div \frac{c}{d}=\frac{a \cdot d}{b \cdot c}\right)$, regra do sanduíche $\left(\frac{a}{b} \div \frac{c}{d}=\left[\frac{\frac{a}{b}}{\frac{c}{d}}\right]=\frac{a d}{b c}\right)$ e "XX" ( $\left(\frac{a}{b}\right)$ (FLORES, 2008; GARCÍA, 2013).

A análise de 32 sites educativos mostrou que praticamente todos (94\%) usavam o IM para calcular diretamente o resultado de expressões (CHEN et al., 2013). Tal predomínio também é detectado nos estudos sobre conhecimento docente. A pesquisa de García (2013) constatou que, embora o grupo investigado conhecesse ao menos dois algoritmos, a maioria dos sujeitos aceitava o IM e o ID como válidos sem necessidade de justificativa, alegando que é algo que está em livros didáticos.

A maioria associou o ID com o processo de comparar frações e a generalidade do DND não era aceita pela maioria do grupo. Outros estudos mostram que classificar este último algoritmo como um procedimento incorreto é um erro comum entre professores e licenciandos que, por vezes, também desconsideram que o conceito de frações equivalentes lhe dá suporte nos casos em que $a$ não é múltiplo de $c$ ou $b$ não é de $d$ (GARCÍA, 2013; LI; KULM, 2008; ÖZEL, 2013; TIROSH, 2000). Nestes casos o DND não vai resultar em uma fração do tipo inteiro sobre inteiro, mas sim resultará em uma fração complexa e, por isso, exigirá outros conceitos para a conclusão da divisão. Estes resultados parecem apoiar o uso de tarefas formativas que levem os sujeitos a refletir sobre o porquê da divisão de frações, isto é, sobre justificativas de algoritmos (BARBOSA, 2011; KILPATRICK; SWAFFORD; FINDELL, 2001; OLANOFF, 2011; TIROSH, 2000).

Identificamos nas produções do corpus duas abordagens, de naturezas distintas, usadas para justificar algoritmos. A primeira delas se destina à legitimação estritamente matemática, por meio de justificativas algébricas para os algoritmos IM (LI, 2008; LOPES, 2008; ÖZEL, 2013; PAYNE, 1976; TIROSH, 2000), ID (LI, 2008; PAYNE, 1976; SILVA; ALMOULOUD, 2008) e DND (SILVA; ALMOULOUD, 2008; TIROSH, 2000), conforme Quadro 1. Esses modos de justificar são importantes para que o (futuro) professor conheça o porquê se procede de tal modo. Estudos sobre conhecimento docente mostram que (futuros) professores cometem o erro de considerar o DND como incorreto e não saber explicar por que ele funciona (GARCÍA, 2013; LI; KULM, 2008; ÖZEL, 2013; TIROSH, 2000). 
É comum que (futuros) docentes tentem avaliar a generalidade/validade de um algoritmo por meio de exemplos ou contraexemplos - como em Salinas (2009) e Tirosh (2000) com o DND -, ao invés de trocar números por variáveis. Apenas um de 17 professores usou argumentação formal (algébrica) para justificar a validade dos algoritmos IM, ID e DND (GARCÍA, 2013). Esta diferença parece estar ligada à limitação de conhecimento matemático (cf. descrito anteriormente) e ao fato de as abordagens numéricas serem consideradas mais acessíveis para alunos jovens (TIROSH, 2000). Ainda assim, é importante que docentes construam conhecimento de justificativas algébricas de um algoritmo, pois ele pode ajudar na tarefa de avaliar os métodos intuitivos elaborados por estudantes (SALINAS, 2009).

\section{ALgORITMO INVERTER E MULTIPLICAR (IM)}

J1) $\frac{a}{b} \div \frac{c}{d}=\frac{\frac{a}{b}}{\frac{b}{d}}=\frac{\frac{a}{b} \times \frac{d}{c}}{\frac{c}{d} \times \frac{d}{c}}=\frac{a}{b} \times \frac{d}{c}=\frac{a}{b} \times \frac{d}{c}=\frac{a d}{b c}$

J2) $\frac{a}{b} \div \frac{c}{d}=K \Rightarrow K \times \frac{c}{d}=\frac{a}{b} \Rightarrow\left(K \times \frac{c}{d}\right) \times \frac{d}{c}=\frac{a}{b} \times \frac{d}{c} \Rightarrow K \times\left(\frac{c}{d} \times \frac{d}{c}\right)=\frac{a}{b} \times \frac{d}{c} \Rightarrow$ $K \times 1=\frac{a}{b} \times \frac{d}{c} \Rightarrow K=\frac{a}{b} \times \frac{d}{c} \Rightarrow K=\frac{a d}{b c}$

J3) Se o inverso de $\frac{c}{d}$ é $\frac{1}{\frac{c}{d}}=\frac{d}{c}$, então $\frac{a}{b} \div \frac{c}{d}=\frac{\frac{a}{c}}{\frac{b}{d}}=\frac{a}{b} \times \frac{1}{\frac{c}{d}}=\frac{a}{b} \times \frac{d}{c}=\frac{a d}{b c}$

J4) Se $\frac{a}{b}$ é equivalente a $\frac{a c d}{b c d}$, então $\frac{a}{b} \div \frac{c}{d}=\frac{a c d}{b c d} \div \frac{c}{d}=\frac{a c d \div c}{b c d \div d}=\frac{a d}{b c}$

ALGORITMO Dividir NUMERADORES E DENOMINADORES ENTRE SI (DND)

J5) $\frac{a}{b} \div \frac{c}{d}=\frac{a}{b} \times \frac{d}{c}=\frac{a \times d}{b \times c}=\frac{a \times d}{c \times b}=\frac{a}{c} \times \frac{d}{b}=\frac{a}{c} \div \frac{b}{d}=\frac{a \div c}{b \div d}$

\section{ALGORITMO IGUALAR DENOMINADORES (ID)}

J6) $\frac{a}{b} \div \frac{c}{d}=\frac{a d}{b d} \div \frac{b c}{b d}$ é igual a $\frac{b d \times a d}{b d} \div \frac{b d \times b c}{b d}=\frac{a d}{1} \div \frac{b c}{1}=a d \div b c=\frac{a d}{b c}$ ou igual a $\frac{a d}{b d} \times \frac{b d}{b c}=\frac{a d}{b c}$

Quadro 1 - Justificativas algébricas de algoritmos da divisão de frações

Fonte: Dados organizados pelo autor a partir do corpus de estudos revisado (2019).

A segunda abordagem se destina a construir uma interação produtiva com crianças (alunos do Ensino Fundamental) para justificar a elas o porquê se inverte-e-multiplica. Tratase da argumentação construída a partir dos conceitos matemáticos que alunos já estudaram até quarto, quinto ou sexto anos (como propriedades da divisão, frações equivalentes e expressões numéricas) e que utiliza estrutura da justificativa algébrica $\mathrm{J} 1$, porém com exemplos numéricos: $\frac{4}{5} \div \frac{2}{3}$ (LI, 2008) e $\frac{3}{5} \div \frac{2}{7}$ (BARBOSA, 2011). Embora as justificativas aritméticas sejam meras verificações se tomadas no campo da produção de conhecimento do matemático, para o docente da Educação Básica elas envolvem conhecimento, tanto no domínio matemático (a exemplo da justificativa algébrica do algoritmo em si), quanto no didático do conteúdo, relativo ao nível de desenvolvimento conceitual e procedimental dos alunos que 
permita a compreensão naquela etapa escolar (quarto a sexto anos) do porquê se inverte-emultiplica (por meio de argumentação com números, ao invés de incógnitas).

Em diversos trabalhos encontramos procedimentos alternativos aos algoritmos para fazer a divisão de frações. Trata-se da manipulação de diagramas pictóricos, incluindo formas geométricas (retangulares, circulares, poligonais ou lineares) ou imagens de objetos. Alguns estudos chamam isto simplesmente de resolver com desenho (NILLAS, 2003; RULE; HALLAGAN, 2006). Eles puderam ser classificados em dois tipos, segundo a ação que lhe edifica: 1. transformar a divisão de frações em divisão de inteiros (CHEN et al., 2013; CONTRERAS, 2012; GUERRA; SILVA, 2008; LI, 2008; LI; CHEN; AN, 2009; LIMA, 1983; SÁ, 2015; SHARP; ADAMS, 2002; SILVA; ALMOULOUD, 2008); 2. comparar as partes fracionadas considerando o divisor como unidade principal (CHEN et al., 2013; FLORES, 2013; LI, 2008; PHILIPP, 2000). No Quadro 2 exemplificamos os referidos tipos.

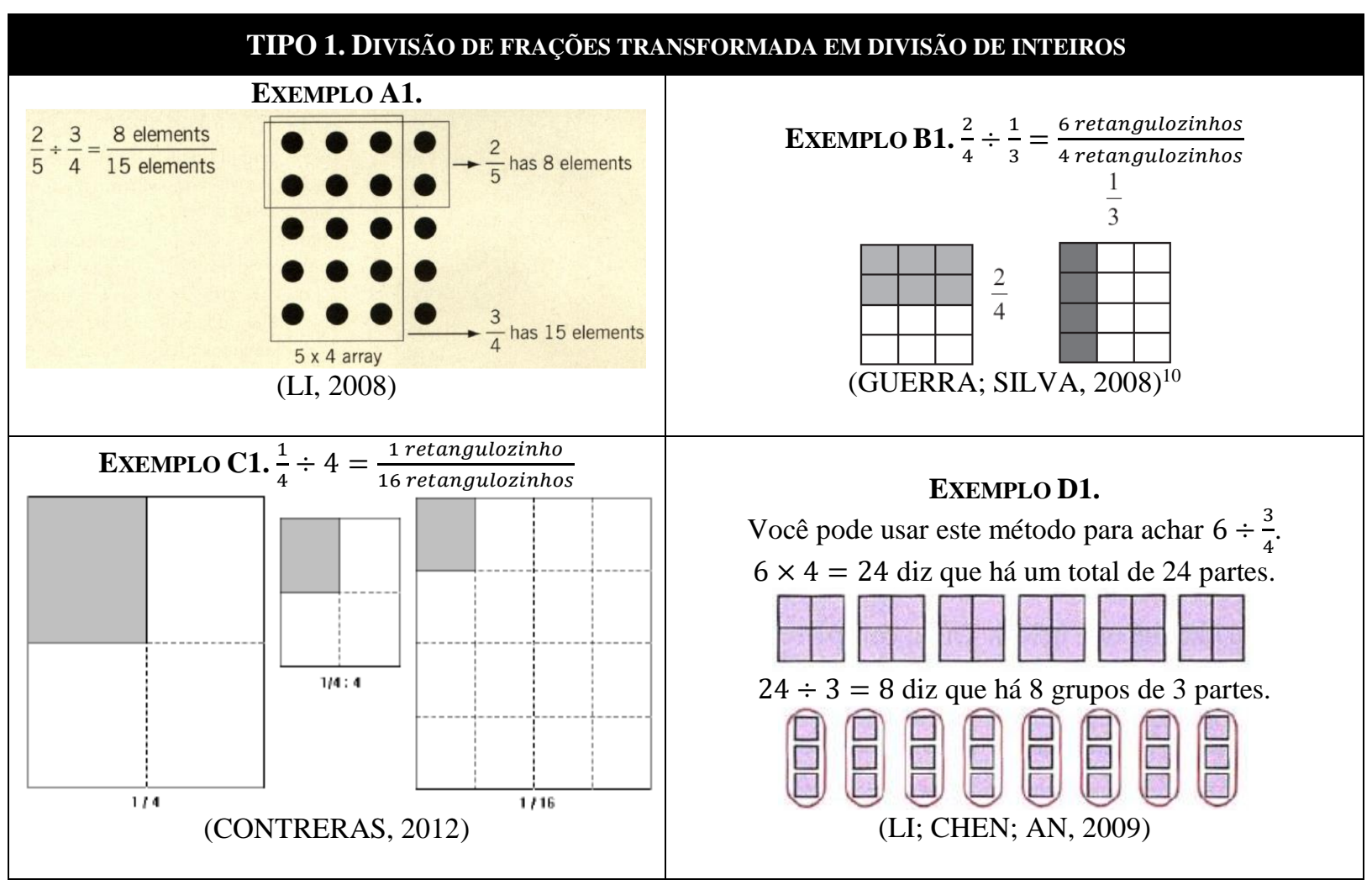

${ }^{10}$ Justifica o procedimento considerando que $\left(\frac{a}{b}\right) \div\left(\frac{c}{d}\right)=m\left(\frac{1}{p}\right) \div n\left(\frac{1}{p}\right)=\frac{m}{n}$, onde $\frac{1}{p}$ é a unidade comum entre dividendo e divisor, cujas quantidades inteiras $m$ e $n$ são, respectivamente, numerador e denominador do quociente que se busca. 


\section{TIPO 2. COMPARAÇÃo DE PARTES FRACIONADAS CONSIDERANDO O DIVISOR COMO UNIDADE PRINCIPAL}

$$
\begin{gathered}
\text { EXEMPLO A2. } \\
1 \frac{3}{5} \div \frac{2}{5}=4
\end{gathered}
$$

(CHEN et al., 2013)

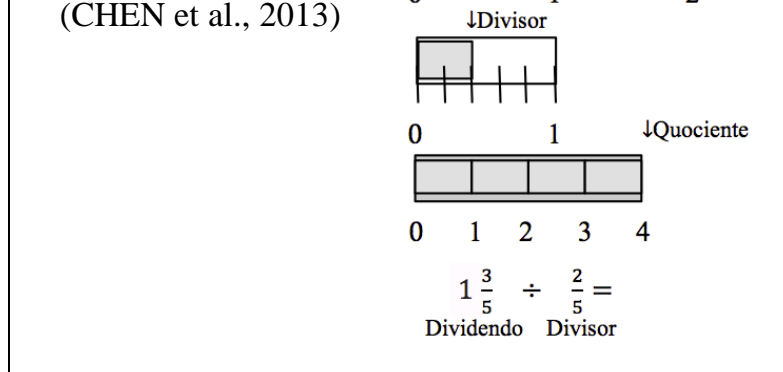

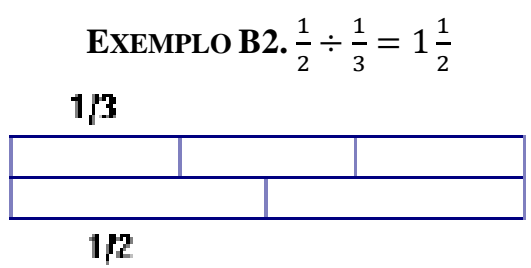

(CONTRERAS, 2012)

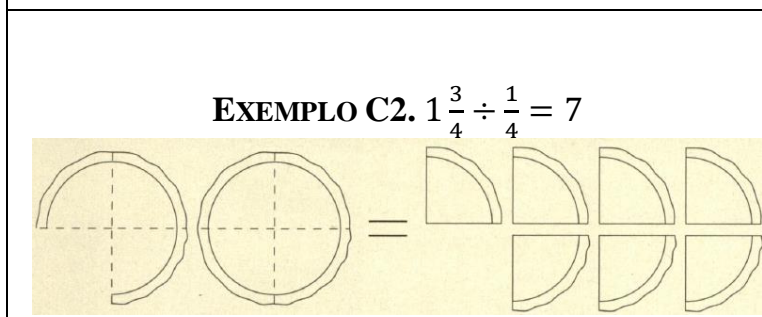

(LI, 2008)

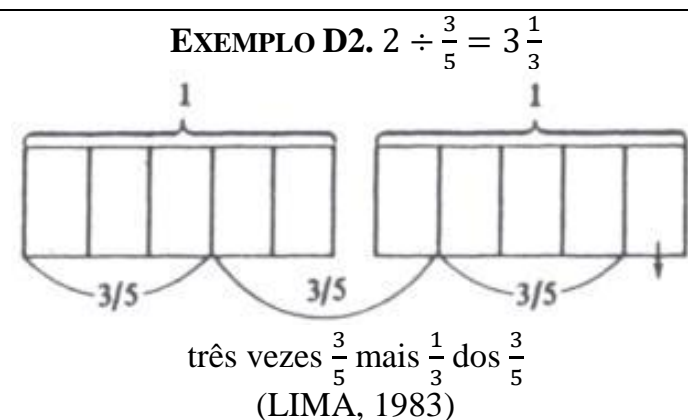

Quadro 2 - Tipos de procedimentos alternativos para divisão de frações e exemplos Fonte: Dados organizados pelo autor a partir do corpus de estudos revisado (2019).

Estudos sobre conhecimento docente mostram a importância de conhecer tais procedimentos, suas justificativas e modos de utilizá-los. Em primeiro lugar, porque eles possibilitam compreender, expressar e ampliar o significado da divisão de frações que professores precisam ter para ensinar (BALL, 1990; LI; KULM, 2008; LO; MCCRORY, 2010; MA, 1999; ÖZEL, 2013). Neste sentido, Lubinski, Fox e Thomason (1998) descreve o percurso de uma licencianda rumo ao desenvolvimento de uma compreensão profunda da divisão de frações, marcado por diversas tentativas de representar pictoricamente tal operação com o intuito de compreender seu significado, culminando na construção de um procedimento particular usando retângulos e círculos, semelhante ao Exemplo A1 no Quadro 2.

Em segundo lugar, tais procedimentos são particularmente úteis para resolver problemas contextualizados (KRIBS-ZALETA, 2006; LO; LUO, 2012; NILLAS, 2003; TIROSH, 2000). Geralmente, os professores têm dificuldade em utilizar tais procedimentos ou mesmo desconhecimento. Em Ball (1990) a minoria dos sujeitos gerou representações circulares adequadas (em forma de pizza) e a metade foi incapaz de gerar alguma. Um licenciando não conseguiu usar um modo diferente do IM para dividir 4 por meio e o outro utilizou inadequadamente uma representação gráfica, em Silveira e Silva (2013).

O melhor cenário é visto em Isiksal e Cakiroglu (2007), no qual boa parte dos sujeitos usou procedimentos pictóricos (circular e retangular, predominando este último), sendo que 
foi mais fácil representar situações em que o dividendo é maior que o divisor, do que ao contrário. Melhoras significativas neste aspecto do conhecimento dos sujeitos foram obtidas em contextos formativos que exploraram diagramas pictóricos ou físicos (GREEN; PIEL; FLOWERS, 2008; RULE; HALLAGAN, 2006; SHARON; SWARTHOUT, 2014).

\subsection{Interpretações e problemas associados}

Entendemos como interpretações da divisão de frações os diferentes tipos de problemas e modelos instrucionais que podem ser categorizados como situações de divisão de frações, nas quais se pode explorar os algoritmos desta operação (SINICROPE; MICK; KOLB, 2002). No corpus investigado elas também são denominadas de estrutura ou classificação de problemas, sentidos de uso ou modelos semânticos. Elas surgem por extensão, sem se limitar, às três interpretações de divisão de números inteiros: partição (determinar o tamanho de cada grupo), medida (determinar a quantidade de grupos) e inverso do produto cartesiano (determinar uma das dimensões de uma figura retangular).

A análise da variedade de nomenclaturas e descrições das interpretações encontradas no corpus, nos levou a agrupá-las (Quadro 3) em torno de uma característica-chave que as distinguissem, não apenas pelo significado da divisão com frações involucrado, mas também considerando os tipos, a quantidade de grandezas ${ }^{11}$ envolvidas e como se relacionam, aspectos estes considerados essenciais (FLORES, 2008; PIEL; GREEN, 1994) e que estavam ausentes na maioria das nomenclaturas revisadas. Acreditamos que as seis características-chave de problemas de divisão de frações criadas aqui unificam as contribuições dos estudos revisados e permitem avançar em termos de funcionalidade nas atividades de classificar e criar problemas para o ensino de divisão de frações. Algo relevante considerando que as investigações sobre o conhecimento docente relacionado a problemas evidenciam dificuldades dos sujeitos em criá-los (CHINNAPPAN; DESPLAT, 2012; GARCÍA, 2013; NILLAS, 2003; ÖZEL, 2013; REDMOND, 2009; SHARON; SWARTHOUT, 2014).

\begin{tabular}{|c|c|}
\hline $\begin{array}{c}\text { NOMENCLATURAS EQUIVALENTES } \\
\text { DAS INTERPRETAÇÕES }\end{array}$ & CARACTERÍSTICA-CHAVE \\
\hline $\begin{array}{l}\text { Partição }^{1} ; \text { Partição com foco em compartilhar ou dividir } \\
\text { igualmente }{ }^{2} ; \text { Compartilhamento }\end{array}$ & 1. Distribuição por inteiros \\
\hline $\begin{array}{c}\text { Medida, cotição ou subtração repetida }{ }^{4} \text { Empacotamento }^{5} ; \text { Divisão } \\
\text { medição por comparação }\end{array}$ & 2. Comparação em uma grandeza \\
\hline $\begin{array}{l}\text { Inverso da multiplicação }{ }^{7} \text {; Razão unitária }{ }^{8} \text {; Inversão do fator } \\
\text { multiplicativo }{ }^{9} \text { Divisão partição por comparação }{ }^{10}\end{array}$ & $\begin{array}{l}\text { 3. Transformação de uma grandeza } \\
\text { dada uma razão adimensional }{ }^{21}\end{array}$ \\
\hline Proporção de valor unitário conhecido ${ }^{11}$; Divisão medição & 4. Proporção entre duas grandezas com \\
\hline
\end{tabular}

\footnotetext{
${ }^{11}$ Entendemos grandeza como sendo tudo que pode ser medido, como comprimento, tempo, peso, área, etc.
} 


\begin{tabular}{|c|c|}
\hline $\begin{array}{l}\text { NOMENCLATURAS EQUIVALENTES } \\
\text { DAS INTERPRETAÇÕES }\end{array}$ & CARACTERÍSTICA-CHAVE \\
\hline equitativa $^{12}$ & um valor unitário conhecido \\
\hline $\begin{array}{l}\text { Determinação da razão unitária }^{13} \text {; Divisão partição equitativa }{ }^{14} \text {; } \\
\text { Proporção de valor unitário desconhecido }\end{array}$ & $\begin{array}{l}\text { 5. Proporção entre duas grandezas em } \\
\text { busca do valor unitário desconhecido }\end{array}$ \\
\hline $\begin{array}{l}\text { Divisão como inverso do produto cartesiano }{ }^{16} \text {; Divisão de área } \\
\text { retangular }{ }^{17} \text {; Produto e fatores }{ }^{18} \text {; Inverso do produto cartesiano } \\
\left.(\text { área })^{19} \text {; Inversão da multiplicação (ou fator perdido) }\right)^{20}\end{array}$ & $\begin{array}{l}\text { 6. Um fator desconhecido de um } \\
\text { produto (área retangular) }\end{array}$ \\
\hline
\end{tabular}

Quadro 3 - Característica-chave de problemas de divisão de frações e respectiva equivalência entre nomenclaturas das interpretações de divisão de frações

Fonte: Dados organizados pelo autor a partir do corpus analisado (2019)

Notas. 1. (OLANOFF, 2011; ÖZEL, 2013; CONTRERAS, 2012); 2. (SINICROPE; MICK; KOLB, 2002; NILLAS, 2013); 3. (PURITZ, 2005); 4. (SINICROPE; MICK; KOLB, 2002; OLANOFF, 2011; ÖZEL, 2013; NILLAS, 2013; CONTRERAS, 2012); 5. (PURITZ, 2005); 6. (LO; LUO, 2012); 7. (SINICROPE; MICK; KOLB, 2002); 8. (NILLAS, 2013); 9. (CONTRERAS, 2012); 10. (LO; LUO, 2012); 11. (CONTRERAS, 2012); 12. (LO; LUO, 2012); 13. (SINICROPE; MICK; KOLB, 2002); 14. (LO; LUO, 2012); 15. (CONTRERAS, 2012); 16. (SINICROPE; MICK; KOLB, 2002); 17. (LO; LUO, 2012); 18. (OLANOFF, 2011; MA, 1999 ); 19.

(NILLAS, 2013); 20. (CONTRERAS, 2012); 21. É a comparação entre duas quantidades de uma mesma grandeza por meio do quociente entre elas, expressa por um número simples sem dimensão.

Passamos a descrever cada uma das referidas interpretações (sintetizadas pelas características-chave), cujos exemplos associados se apresentam no Quadro 4. A primeira consiste na distribuição de valor fracionário entre inteiros, em determinar o tamanho de cada uma das partes quando se sabe o número de partes (inteiro). A segunda trata de comparar dois valores de uma mesma unidade de medida escolhendo uma delas para ser a unidade de referência, o resultado indicará quanto o dividendo contém tal unidade (o divisor), podendo ser fração própria ou imprópria. Quando o dividendo é maior que o divisor, a solução pode ser também por subtração repetida (OLANOFF, 2011) ou adição repetida (NILLAS, 2003).

\begin{tabular}{|c|c|c|c|}
\hline $\begin{array}{c}\text { CARACTERÍSTICA- } \\
\text { CHAVE }\end{array}$ & EXEMPLOS DE PROBLEMAS ASSOCIADOS & $\begin{array}{c}\text { SOLUÇÃO } \\
\text { [UNIDADE] }\end{array}$ & $\begin{array}{c}\text { ANÁLISE DE } \\
\text { UNIDADES }\end{array}$ \\
\hline $\begin{array}{l}\text { 1. Distribuição por } \\
\text { inteiros }\end{array}$ & $\begin{array}{l}\text { - Se } \frac{3}{7} \text { de um bolo foi repartido entre } 5 \text { crianças, } \\
\text { quanto do bolo terá cada uma delas? } \\
\text { - Repartir } \frac{3}{7} \text { de um saco de balas entre } 5 \text { crianças. }\end{array}$ & $\begin{array}{c}\frac{3}{7} \div 5 \\
{[\text { do bolo }]} \\
{[\text { do saco }]}\end{array}$ & $\begin{array}{c}\text { Dividendo e } \\
\text { quociente tem } \\
\text { mesma unidade, } \\
\text { divisor inteiro }\end{array}$ \\
\hline $\begin{array}{l}\text { 2. Comparação em } \\
\text { uma grandeza }\end{array}$ & $\begin{array}{l}\text { - Quantas vezes } \frac{1}{4} \mathrm{~kg} \text { de farinha cabe em } 5 \frac{1}{2} \mathrm{~kg} \text { de } \\
\text { farinha? } \\
\text { - Hoje me exercitei } \frac{1}{4} \text { de hora e ontem } 1 \frac{1}{2} \text { hora. } \\
\text { Quantas vezes me exercitei hoje comparado a ontem? }\end{array}$ & $\begin{array}{l}5 \frac{1}{2} \div \frac{1}{4} \\
\text { [vezes] } \\
\frac{1}{4} \div 1 \frac{1}{2} \\
\text { [vezes] }\end{array}$ & $\begin{array}{l}\text { Dividendo e } \\
\text { divisor tem } \\
\text { mesma unidade }\end{array}$ \\
\hline $\begin{array}{l}\text { 3. Transformação } \\
\text { de uma grandeza, } \\
\text { sabendo uma razão } \\
\text { adimensional } \\
\text { (A em B dado A/B) }\end{array}$ & $\begin{array}{l}\text { - Qual é o tamanho original de um elástico que } \\
\text { esticado mede } 1 \frac{1}{2} \mathrm{~m} \text {, sabendo que ele pode ser } \\
\text { esticado até } 4 \frac{1}{4} \text { vezes seu tamanho? } \\
\text { - João correu } 1 \frac{1}{2} \mathrm{~km} \text { ontem e isto é } \frac{3}{8} \text { da sua meta, } \\
\text { assim sendo, quantos quilômetros ele planeja correr? }\end{array}$ & $\begin{array}{l}1 \frac{1}{2} \div 4 \frac{1}{4} \\
{[\text { metro] }} \\
1 \frac{1}{2} \div \frac{3}{8} \\
{[\mathrm{~km}]}\end{array}$ & $\begin{array}{l}\text { Dividendo e } \\
\text { quociente tem } \\
\text { mesma unidade, } \\
\text { divisor é razão } \\
\text { adimensional }\end{array}$ \\
\hline $\begin{array}{l}\text { 4. Proporção entre } \\
\text { duas grandezas com } \\
\text { um valor unitário } \\
\text { conhecido }\end{array}$ & $\begin{array}{l}\text { - Quantos pacotes eu posso fazer usando } 5 \frac{1}{2} \mathrm{~kg} \text { de } \\
\text { farinha sabendo que cabe } \frac{1}{4} \mathrm{~kg} \text { em um pacote? } \\
\text { - Quanto tempo um barco demora para percorrer } 5 \frac{1}{2} 2 \\
\mathrm{~km} \text { indo a } 71 / 2 \mathrm{~km} \text { por hora? }\end{array}$ & $\begin{array}{l}5 \frac{1}{2} \div \frac{1}{4} \\
\text { [pacotes] } \\
5 \frac{1}{2} \div 7 \frac{1}{2} \\
\text { [hora] }\end{array}$ & $\begin{array}{l}\text { Todas unidades } \\
\text { são diferentes, } \\
\text { divisor é valor } \\
\text { unitário entre } \\
\text { duas unidades }\end{array}$ \\
\hline $\begin{array}{l}\text { 5. Proporção entre } \\
\text { duas grandezas em } \\
\text { busca do valor } \\
\text { unitário }\end{array}$ & $\begin{array}{l}\text { - Quantos quilômetros o carro percorre com } 1 \text { tanque } \\
\text { de combustível, em se } 10 \frac{1}{2} \mathrm{~km} \text { ele usa } \frac{3}{4} \text { de tanque? }\end{array}$ & $\begin{array}{l}10 \frac{1}{2} \div \frac{3}{4} \\
{[\mathrm{~km} \text { por }} \\
\text { tanque] }\end{array}$ & $\begin{array}{l}\text { Todas unidades } \\
\text { são diferentes, } \\
\text { quociente é } \\
\text { valor unitário }\end{array}$ \\
\hline
\end{tabular}




\begin{tabular}{|cccc|}
\hline $\begin{array}{c}\text { CARACTERístiCA- } \\
\text { CHAVE }\end{array}$ & EXEMPlos DE PROBLEMAS ASSOCIADOS & $\begin{array}{c}\text { SolUÇÃO } \\
\text { [UNIDADE] }\end{array}$ & $\begin{array}{c}\text { ANÁLISE DE } \\
\text { UNIDADES }\end{array}$ \\
\hline desconhecido & $\begin{array}{c}\text { - Quantos centímetros mede uma polegada, sabendo } \\
\text { que } 2 \frac{1}{2} \text { polegadas medem aproximadamente } 6 \frac{1}{3} \mathrm{~cm} ?\end{array}$ & $\begin{array}{c}6 \frac{1}{3} \div 2 \frac{1}{2} \\
\text { [cm por pol.] }\end{array}$ & $\begin{array}{c}\text { entre as } \\
\text { unidades }\end{array}$ \\
\hline $\begin{array}{c}\text { 6. Um fator } \\
\text { desconhecido de um } \\
\text { produto (área } \\
\text { retangular) }\end{array}$ & - Se a área de um retângulo tem $3 \frac{3}{4} \mathrm{~m}^{2}$ e sua largura & Dividendo e \\
horta retangular de largura $1 \frac{1}{2}$ metros e área $3 \frac{3}{4} \mathrm{~m}^{2} ?$ & $3 \frac{3}{4} \div 1 \frac{1}{2}$ & $\begin{array}{c}\text { quociente tem } \\
\text { unidades } \\
\text { diferentes }\end{array}$ \\
\hline
\end{tabular}

Quadro 4 - Interpretações para divisão de frações, exemplos de problemas, soluções e análise de unidades Fonte: Dados organizados pelo autor a partir do corpus de estudos revisado (2019).

A terceira diz respeito à transformação de uma quantidade A de uma grandeza (ou o valor de uma parte de um todo) sabendo a razão adimensional $A / B$ entre a medida $A$ e uma medida B (ou entre os valores da parte e do todo) para obter o valor da medida B (ou do todo) na mesma unidade de A. Isto se traduz em "fazer o dividendo tantas vezes menor quanto indica o numerador do divisor e tantas vezes maior quanto indica o denominador do divisor" (CONTRERAS, 2012, p. 84, tradução nossa). A ideia de operador é um fundamento desta interpretação, mas ela se diferencia de problemas de multiplicação porque nestes a razão dada é B/A (ou do todo pela parte) ao invés de ser A/B. A quarta consiste em calcular a proporção entre duas grandezas diferentes, sendo uma delas o valor unitário entre as grandezas. Cabe determinar uma quantidade (Q) de uma grandeza associada a uma quantidade dada (D) de outra grandeza quando se conhece o valor unitário da segunda em relação à primeira $\left(\frac{d}{1}\right)$.

A quinta diz respeito ao uso da proporção entre duas grandezas diferentes como meio de determinar o valor unitário desconhecido de uma em relação a outra. Trata-se de encontrar o valor unitário $(\mathrm{Q})$ a partir da relação entre duas quantidades de duas grandezas distintas $\left(\frac{D}{d}\right)$. Problemas que envolvam o cálculo de velocidade, densidade ou preço unitário são exemplos deste modelo. A sexta é muito associada a problemas de área retangular, pois consiste em calcular um fator desconhecido de um produto entre dois fatores, ou seja, obter uma das medidas $(\mathrm{m})$ quando se conhece a outra $(\mathrm{M})$ e o produto delas $(P=m . M)$.

Exemplos apresentados nas características-chave 2 e 4 do quadro anterior mostram que um mesmo contexto (envolvendo divisão e farinha) pode gerar a redação de problemas diferentes e enfatizar interpretações distintas da divisão de frações. Para reforçar tal diferença, complementamos as características-chave com a análise das unidades envolvidas nos respectivos problemas, a qual permite compreender melhor a natureza dos mesmos (FLORES, 2008). No caso em questão, o problema de comparação em uma grandeza possui unidades diferentes no dividendo ( $\mathrm{kg}$ de farinha) e no quociente (quantidade de vezes), enquanto no problema de proporção entre duas grandezas com um valor unitário conhecido todas as 
unidades são diferentes entre si, sendo que a do divisor ( $\mathrm{kg} /$ pacote) é o valor unitário dado em função das unidades do dividendo $(\mathrm{kg})$ e do quociente (pacote). Outro exemplo de redações diferentes ocorre em contexto de alargamento de elástico, contemplando a transformação em uma grandeza dada uma razão adimensional (cf. quadro anterior) e a comparação em uma grandeza: "um elástico medindo $\frac{1}{4}$ metro pode esticar até $1 \frac{1}{2} \mathrm{~m}$. Qual é o fator de alargamento?" (FLORES, 2008, p. 3, tradução nossa).

Os estudos sobre o conhecimento docente relacionado às interpretações mostram que são duas as mais comumente conhecidas pelos sujeitos: partição (característica-chave 1) e medida (característica-chave 2) (BALL, 1990; ISIKSAL; CAKIROGLU, 2007; KRIBSZALETA, 2006; LO; LUO, 2012; LUBINSKI; FOX; THOMASON, 1998; TIROSH, 2000).

Em situações de divisão de frações por número inteiro, a maioria dos sujeitos do corpus interpretara corretamente utilizando a ideia de distribuição, partição conforme característica-chave 1 (BALL, 1990; ISIKSAL; CAKIROGLU, 2007; LO; LUO, 2012). Quando o divisor também é uma fração, torna-se mais natural e bem sucedido o uso da ideia de medida ou comparação de dois valores em uma mesma grandeza conforme a característicachave 2 (BALL, 1990; LO; LUO, 2012; LUBINSKI; FOX; THOMASON, 1998; PURITZ, 2005). Embora esta interpretação seja mais facilmente estendida do conjunto dos naturais para as frações do que a primeira (OLANOFF, 2011), é comum que professores ou licenciandos obtenham êxito nesta extensão em casos com dividendo maior que o divisor e insucesso nos casos contrários (ISIKSAL; CAKIROGLU, 2007).

A elaboração de problemas de divisão de frações não é uma tarefa simples, professores formados foram melhores em resolver um problema do que em elaborar ou associar um contexto a uma expressão dada (LO; LUO, 2012). Além disso, a quantidade de anos na docência por si só parece não contribuir para o desenvolvimento de um corpo de conhecimentos específicos para este tipo de atividade seja suficiente para distinguir experientes de iniciantes (CHINNAPPAN; DESPLAT, 2012).

Parte desta deficiência está associada à diferenciação entre problemas de divisão e de multiplicação de frações que, por si só, não são tarefas elementares. A estrutura de corpo algébrico do conjunto dos números racionais é caracterizada pela estrutura multiplicativa, o que possibilita a divisão de frações ser construída como o inverso da multiplicação como vimos anteriormente. Assim, "um problema não é de multiplicar ou de dividir [frações] por sua própria estrutura (que é a mesma), mas sim pelos dados que dispomos, pela forma de 
resolvê-lo" (FLORES, 2008, p. 1) e, incluímos, pela relação entre as grandezas envolvidas e a pergunta que se quer responder.

Nos estudos em que houve resoluções bem sucedidas de problemas contextualizados por licenciandos e professores, embora esta seja uma tarefa desafiadora para os primeiros (NILLAS, 2003), identificou-se uso frequente de representações pictóricas, além de geométricas, aritméticas e tabulares (ISIKSAL; CAKIROGLU, 2007; LO; LUO, 2012; NILLAS, 2003; TIROSH, 2000).

Uma investigação sobre as estratégias inventadas por alunos, licenciandos e professores (KRIBS-ZALETA, 2006) mostrou que resolver por desenho é a primeira abordagem escolhida, sendo que a maioria dos investigados resolveu o problema de dividir duas laranjas e meia em porções de três quartos usando o procedimento pictórico do Tipo 1 (similar a D1, cf. Quadro 2) em duas etapas: primeiro, dividiram as duas laranjas e meia em quartos e depois fizeram grupos de 3 quartos, ou seja, eles primeiro multiplicaram a quantidade de laranjas pelo denominador do divisor $\left(2 \frac{1}{2} \cdot 4\right.$ gerando 10 e convertendo a unidade para quartos de laranja) e, em seguida, fizeram a divisão/comparação de números inteiros (10:3). Ainda assim, é recorrente a existência de dificuldades por parte tanto de professores, quanto de licenciandos em representar uma divisão de frações com desenhos (ISIKSAL; CAKIROGLU, 2007; LI; KULM, 2008; LUO; LO; LEU, 2011; MA, 1999; PIEL; GREEN, 1994; RIZVI; LAWSON, 2007), sendo comum confundirem a divisão por $1 \frac{1}{2}$ como sendo metade de, ou seja, divisão por 2 ou multiplicação por 1/2 (BALL, 1990; CHINNAPPAN; DESPLAT, 2012; FLORES, 2008; NILLAS, 2003; RULE; HALLAGAN, 2006; SHARON; SWARTHOUT, 2014).

Estudos que analisaram conexões entre interpretações, problemas, suas resoluções e algoritmos identificaram que alguns algoritmos são mais adequados e fornecem mais sentido para determinados tipos de problemas de divisão de frações (FLORES, 2008; SINICROPE; MICK; KOLB, 2002). Eles também mostram como uma solução intuitiva ou com desenho de um destes problemas pode ser o ponto de partida para desenvolver uma explicação instrucional que sistematize o procedimento da divisão de fração e justifique o algoritmo mais natural para a situação. Constatamos que problemas com as características-chave 2, 3, 5 e 6 se associam aos algoritmos ID, IM, IM e DND, respectivamente. No Quadro 5 apresentamos apenas um exemplo de cada caso, embora existam outras possibilidades de sistematização.

Problemas de Comparação EM UMA grandeza (2) E AlgoritMo IgUALAR DENOMINADORES Problema. Quantas vezes se pode obter $2 / 3$ de bolo tendo 5 bolos? 
restar 1/3 que é a metade da porção (cf. desenho). A expressão se reduz à comparar 15 com 2. Mostrar a lógica de igualar denominadores utilizando o desenho e a expressão aritmética: $5 \div \frac{2}{3}=\frac{15}{3} \div \frac{2}{3}=15 \frac{1}{3} \div 2 \frac{1}{3}=15 \div 2=7 \frac{1}{2}$ (FLORES, 2008).

Problemas de TranSfORMaÇão de UMA GRANDEZA, SABENDO UMA RAZão ADIMENSIONAL (3) E ALGORITMO INVERTER E MULTIPLICAR

Problema. Numa pesquisa com alunos do $7^{\circ}$ ano, 48 disseram preferir pizza. Isto é um vez e meia o número de estudantes que preferem salada. Quantos preferem salada?

Solução possível. $1 \frac{1}{2}=\frac{3}{2}$

$\frac{3}{2} \times$ número para salada $=48$ (se multiplicar

[o número para salada] por 3 e dividir por 2 tem-se 48). Para voltar, multiplique por 2 e divida por 3 , ou multiplique pela fração $2 / 3$. $48 \div 1 \frac{1}{2}=48 \div \frac{3}{2}=48 \times \frac{2}{3}$. 32 preferem salada.

\section{PROBleMA DE PROPORÇÃo ENTRE DUAS GRANDEZAS COM UM VALOR UNITÁRIO DESCONHECIDO (5) E ALGORITMO INVERTER E MULTIPLICAR}

Problema. Uma impressora imprime 20 páginas em 2 minutos e meio. Quantas páginas ela imprime por minuto? Solução possível.

20 páginas em $2 \frac{1}{2}$ minutos

40 páginas em 5 minutos

8 páginas em 1 minutos
Sistematização. Embora diferentes estratégias possam ser usadas para resolver o problema, uma abordagem geral é reverter o procedimento original de multiplicação. Multiplicar por $1 \frac{1}{2}$ equivale a multiplicar por $\frac{3}{2}$ : multiplicando por 3 e dividindo por 2. O reverso é multiplicar por 2 e dividir por 3, ou multiplicar por $\frac{2}{3}$. O processo é simbolicamente representado por $\frac{a}{b} \div \frac{c}{d}=\frac{a}{b} \times \frac{d}{c}$ (SINICROPE; MICK; KOLB, 2002).

\section{PROBLEMA DE UM FATOR DESCONHECIDO DE UM PRODUTO (ÁREA RETANGULAR) E ALGORITMO DIVIDIR}

\section{NUMERADORES E DENOMINADORES ENTRE SI}

Problema. Quanto mede a altura de um retângulo cuja superfície é $1 / 6 \mathrm{~m}^{2}$ e sua base tem $1 / 3 \mathrm{~m}$ ? Solução possível.

$$
\frac{1}{6}: \frac{1}{3}=\frac{1: 1}{6: 3}=\frac{1}{2}
$$

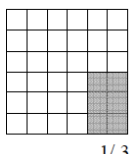

Sistematização. A partir da equação $\frac{1}{3} \cdot x=\frac{1}{6}$, temos que buscar os números que multiplicados pelo numerador e denominador nos dão a nova fração. Para tanto, dividimos numeradores e denominadores (FLORES, 2008).

Quadro 5 - Conexões entre problemas, interpretações e algoritmo para sistematização da divisão de frações Fonte: Flores (2008) e Sinicrope, Mick e Kolb (2002) traduzidos e adaptados pelos autores.

Em relação ao formador de professores, há necessidade de conhecimento de múltiplos modos de representar a divisão de frações e de como eles se relacionam entre si, com as ideias de números inteiros e com os algoritmos. Isto possibilita que ele familiarize os futuros professores com os procedimentos, interpretações e problemas que eles precisarão quando forem ensinar (OLANOFF, 2011). Estudos mostram que com formação específica professores e licenciandos melhoram seu conhecimento sobre estratégias de resolver problemas (usando procedimentos pictóricos e materiais manipulativos) e ampliam a habilidade de elaborar contextos e problemas para expressões de divisão de frações (CHARALAMBOUS; HILL; BALL, 2011; REDMOND, 2009; RULE; HALLAGAN, 2006; TIROSH, 2000).

Estas conclusões estão em consonância com a ideia de que o conhecimento matemático de divisão de frações é uma das bases sob as quais o conhecimento pedagógico de professores é construído (MA, 1999). Isto ganha mais relevância se considerarmos todas as limitações identificadas anteriormente em relação ao conhecimento matemático docente sobre 
divisão de frações e, principalmente, o fato de que muitas vezes o existente é fragmentado (BALL, 1990; CHINNAPPAN; DESPLAT, 2012).

\subsection{Explicações instrucionais, propostas de ensino e recursos didáticos}

Um dos problemas no ensino de divisão de frações é a prescrição de regras e macetes logo no primeiro contato do estudante com a operação (BERTONI, 2008; LOPES, 2008). Isto configura um modo de ensinar baseado na exposição de fórmulas em detrimento do conceito, associado à memorização em detrimento do raciocínio. Trata-se de um tipo de explicação instrucional $^{12}$ não aconselhável, intitulada enunciar a matemática, ou seja, se trata de simplesmente descrever o procedimento, o como fazer (CHARALAMBOUS; HILL; BALL, 2011). Identificou-se a dificuldade de futuros professores superarem este problema e desenvolverem explicações mais adequadas, do tipo ensinar para compreensão, focalizando o significado dos conceitos e procedimentos (CHARALAMBOUS; HILL; BALL, 2011; BAYOUD, 2011). Já os professores em Özel (2013) não souberam fazê-lo em relação ao algoritmo DND. Não surpreende que haja esta limitação no conhecimento didático dos sujeitos, relativa a dar explicações que promovam a compreensão conceitual nos alunos, pois vimos nas seções anteriores que boa parte dos docentes não possui uma compreensão profunda da divisão de frações (MA, 1999), e, mesmo que a tivessem, isto por si só não seria suficiente, pois boas explicações instrucionais

[...] estabelecem a questão central e constroem sobre os conceitos e habilidades
existentes nos alunos, para ajudá-los a se mover do que eles já sabem para o que eles
têm de aprender. Explicações de qualidade também levam em conta dificuldades e
equívocos comuns de alunos, esclarecem o que deve ser aprendido, separam e
sequenciam informações e, também, monitoram o progresso em direção à meta de
aprendizagem. Boas explicações também dependem de escolhas sensatas e uso
cuidadoso de exemplos e representações e tornam transparentes as conexões entre
eles; elas fornecem informações precisas e significativas, bem como, esclarecem a
aplicabilidade das ideias a serem aprendidas. Em matemática, boas explicações
instrucionais também são mais do que simples descrições das etapas envolvidas em
um processo; em vez disso, eles dão significado a essas etapas
(CHARALAMBOUS; HILL; BALL, 2011, p. 443 , tradução nossa).

Diversos estudos convergem para a definição do que é uma boa explicação instrucional, ora complementando, ora aprofundando-a. Os resultados de Barbosa (2011)

12 Termo definido como atividade que inclui "articulações, demonstrações ou arranjos de experiências que professores fazem para comunicar alguma parte da matéria para estudantes e que são explicitamente voltados para apoiar a compreensão do aluno sobre o conteúdo" (CHARALAMBOUS; HILL; BALL, 2011, p. 443, tradução nossa). Em síntese, ela não se restringe a explanações literais e sua função principal é apoiar o estudante na construção de compreensão de um conceito ou procedimento e, portanto, não se trata simplesmente de apresentar o conteúdo. 
confirmam que a produção de explicações para alunos "envolve raciocínio matemático tanto quanto pensamento pedagógico" (p. 11), pois deve considerar aspectos curriculares e ter consciência dos conceitos que os aprendizes já possuem para que haja uma interação produtiva em sala de aula.

O trabalho de Li, Chen e Kulm (2009) investigou o pensamento dos professores na preparação de aulas de divisão de frações e identificou que eles levam em consideração aspectos da aprendizagem do aluno, incluindo: o que já foi aprendido para poder se basear no conhecimento prévio para ensinar o que se quer, bem como abordagens de ensino que possibilitem investigação por parte dos alunos, envolvendo-os ativamente nas atividades. Os resultados de Philipp (2000) indicam três princípios para ensinar para a compreensão: (i) identificar e contemplar os principais conceitos matemáticos do tópico; (ii) construir sobre conhecimento existente dos alunos; (iii) introduzir símbolos e procedimentos depois de contemplados os conceitos que eles representam.

Um estudo sobre a formação docente baseada em atividades de justificativa da divisão de frações a partir da resolução de problemas (LO; MCCRORY, 2010) sugere quatro elementos de conhecimento para um professor ensinar com foco na justificação: (i) conhecer o que conta como justificação válida para uma dada resposta de um problema (o que num contexto do Ensino Fundamental inclui significados dentro do contexto do problema e relações entre as quantidades, representações e expressões envolvidas na explicação); (ii) conhecer dificuldades que estudantes podem ter quando aprendem divisão de frações (e que podem parecer óbvias para o professor); (iii) conhecer como tópicos matemáticos se conectam com operações e sistemas numéricos (a conexão entre um problema de culinária, a justificativa da sua resposta envolvendo divisão de frações, a divisão de números inteiros e o conceito geral de divisão); (iv) conhecer modos de apoiar o raciocínio matemático de alunos, na construção de significados e conexões de conceitos (incluindo questionamentos e colocação de problemas mais simples relevantes).

O corpus revisado possui diversos trabalhos que discutem ou relatam propostas de ensino alinhados com a perspectiva de ensinar para a compreensão. Dentre elas, identificamos abordagens que envolvem sequência de problemas - sejam matemáticos (elementares, geométricos e aritméticos) ou contextualizados (chamados de word problems ou story problems) - ou se baseiam no uso de materiais manipulativos e recursos tecnológicos para levar os estudantes a compreenderem o significado da divisão de frações e sistematizarem (ou generalizarem) uma regra para divisão de frações. A seguir, exemplificamos cada uma delas. 
Abordagem com problemas elementares. Esta proposta consiste em apresentar quantidades pares de frações unitárias sendo divididas por 2 , de modo que alunos desde o $5^{\circ}$ ano conseguem obter a solução realizando divisão de inteiros (BERTONI, 2009). O problema 8 sextos $\div 2$ pode ser interpretado como oito pedaços de um sexto sendo divididos igualmente entre duas crianças, que pode ser entendido como quatro pedaços de um sexto para cada criança, ou $\frac{4}{6}$. Outros problemas seriam 4 quintos $\div 2,6$ terços $\div 2$ e meio $\div 2$ (entendido como metade de meio). Entendemos que se trata de uma abordagem de caráter introdutório e também é especialmente útil quando se necessita fazer uma transformação (simplificação ou analogia) que ajude a explicar a solução de um problema ou resolução por desenho. Por exemplo, calcular a divisão equitativa de 10 décimos de uma pizza entre 5 amigos (SINICROPE; MICK; KOLB, 2002) ou calcular quantas porções de $\frac{2}{3}$ de pizza se obtém com $\frac{5}{3}$ de pizza (dado pela divisão de 5 terços por 2 terços) se reduz a comparar 5 com 2 , resultando $\frac{5}{2}=2 \frac{1}{2}$ porções (FLORES, 2008).

Abordagem com problemas exclusivamente aritméticos. Esta proposta, que também pode ser pensada para o início do tratamento da divisão de frações, consiste em apresentar uma expressão numérica como um problema, sem a prescrição de regras, como um ponto de partida "para processos de descoberta e reinvenção da matemática" (LOPES, 2008, p. 11). O autor constrói esta proposta com base na ideia de que são escassas as possibilidades de abordar intuitivamente a divisão de frações ou com aplicações realistas.

Sua abordagem foi feita com alunos de 12 anos e começou com o problema $\frac{14}{15} \div \frac{2}{5}$. Os alunos começaram naturalmente dividindo numeradores e denominadores entre si (DND) e usaram duas ideias-chave (a divisão como operação inversa da multiplicação e o conceito de frações equivalentes). Após discutirem mais dois exemplos $\left(\frac{3}{14} \div \frac{2}{7} \mathrm{e} \frac{2}{3} \div \frac{5}{7}\right)$ eles generalizaram verbalmente um procedimento de dividir frações (nos moldes de J4, Quadro 1), ainda que não utilizassem linguagem algébrica. Coube ao professor decidir, ao final, se os alunos estavam aptos para compreender a sistematização algébrica da discussão.

Abordagem com problemas geométricos. Esta proposta consiste na iniciação dos estudantes à divisão de frações por meio do processo de contagem de unidades comuns de retângulos que representam as frações (cf. Exemplo B1, no Quadro 2) e, com isto, caminha-se para justificar o algoritmo da divisão (GUERRA; SILVA, 2008). A vantagem dessa proposta é que a "caracterização das operações com frações como um processo de contagem, estrutura já estabelecida no sistema cognitivo da maioria dos alunos, estabelece uma relação com os 
inteiros no sentido em que operar com elas é similar a operar com os inteiros" (GUERRA; SILVA, 2008, p. 51). Entretanto, é preciso ter cautela, pois a correta manipulação de figuras geométricas divididas e pintadas pode gerar a impressão de que o aluno possui um conhecimento conceitual que talvez não tenha (BERTONI, 2009).

Abordagem com problemas aritméticos e geométricos. Trata-se de ensinar a divisão de frações por meio de sequências de atividades exploratórias para os alunos (cf. Quadro 4), fundamentada na ideia de medida ou quantos cabem? (conforme característica-chave 2), propriedades sobre frações e números naturais já conhecidas dos alunos e no algoritmo Dividir Numeradores e Denominadores entre si, considerado uma "regra operatória semelhante à multiplicação" (SILVA; ALMOULOUD, 2008, p. 75). Os autores defendem que o algoritmo Inverter e Multiplicar "pode ser apresentado posteriormente no trabalho com frações algébricas. Antes disso é interessante que se utilize outros processos a fim de construir significado à divisão dos fracionários" (SILVA; ALMOULOUD, 2008, p. 75).

Atividade 1.
a) Quantas metades cabem em um inteiro? Como você
pode representar essa situação?
b) Quantos terços cabem em um inteiro? Como você
pode representar essa situação?

\section{Atividade 3 (ideia de operador).}

Observe os desenhos e complete

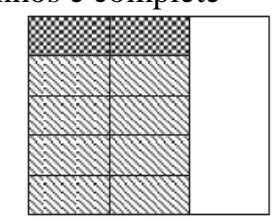

Se um quinto de dois terços é $\frac{1}{5} \times \frac{2}{3}=$

Então podemos escrever que:

$$
\begin{aligned}
& \frac{2}{15} \div \frac{1}{5}= \\
& \frac{2}{15} \div \frac{2}{3}=
\end{aligned}
$$

Atividade 2. Observe os desenhos e complete a)

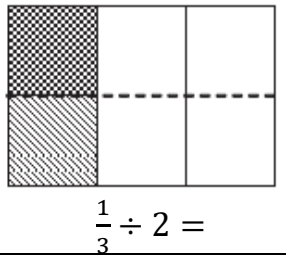

b)

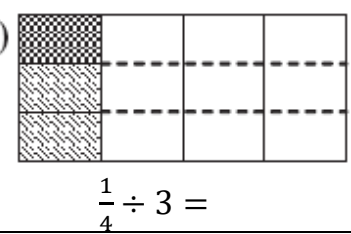

Atividade 4.

Quantos oitavos cabem em 1/16? Dê a expressão matemática que representa a situação. Utilize a figura abaixo para ajudar na solução.

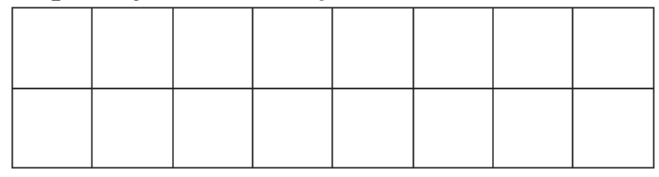

Atividade 5. Quantos $1 / 3$ cabem em $1 / 2$ ? Utilize a figura abaixo para ajudar na solução.

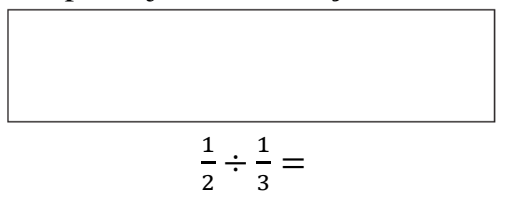

Atividade 6. Escreva uma regra que mostre como dividir duas frações quaisquer Quadro 6 - Abordagem de ensino como sequência de atividades exploratórias Fonte: Silva e Almouloud (2008)

Embora introduzir a divisão de fração por meio de figuras possa não ser tão simples, em virtude da necessidade de visualizar e compreender as partes envolvidas, outros trabalhos também defendem este tipo de abordagem utilizando sequência de problemas e procedimentos pictóricos (LIMA, 1983; SÁ, 2015). 
Abordagem com problemas contextualizados. Atividades instrucionais que envolvam a resolução de problemas de divisão de frações em contextos extra-matemáticos podem ajudar os estudantes a superar ou evitar possíveis equívocos quando são conectados dois tipos de significado (o referencial, derivado de objetos manipulativos, e o quantitativo, relacionado aos cálculos aritméticos). Isto permite aos alunos combinar conhecimento intuitivo e procedimentos computacionais simbólicos (PIEL; GREEN, 1994). Por exemplo, o problema ' $1 / 2$ pizza dividido por $1 / 4$ de pizza' permite perceber o significado quantitativo obtido pelo resultado do algoritmo inverter e multiplicar (igual a 2), e também enfatiza o significado referencial (não significa 2 pizzas inteiras, mas sim a quantidade de quartos de pizza que cabem em meia pizza) de modo a não substituir o significado da divisão (comparação) pela da multiplicação.

Nesta perspectiva, a proposta de Hiratsuka (1996) sugere a colocação de uma série de problemas (de proporção de valor unitário desconhecido) relacionando duas variáveis (dinheiro e balas, latas de tinta e paredes etc.) para que os alunos resolvam intuitivamente (com a ideia de divisão de naturais) e sejam levados, gradativamente, à justificativa do aparecimento dos produtos cruzados, percebendo assim o inverter-e-multiplicar. São eles: $1^{\circ}$ ) Se com dois reais compro quatro balas, quantas balas comprarei com um real? $2^{\circ}$ ) Se com 2 latas de tinta pinto 6 paredes, quantas paredes eu pintarei com 1 lata de tinta? $3^{\circ}$ ) Se com $\frac{1}{2}$ lata de tinta eu pinto 1 parede, quantas paredes eu pintarei com 1 lata de tinta? $4^{\circ}$ ) Se com $\frac{2}{3}$ de uma lata dá pra pintar $\frac{3}{4}$ de uma parede, que fração da parede pintarei com 1 lata de tinta?

Ainda nesta abordagem, a proposta de Li (2008) está alinhada à explicação conceitual presente nos livros didáticos chineses (LI; CHEN; AN, 2009) e visa derivar uma regra computacional a partir da solução de problemas com o uso de representações pictóricas. Primeiro trabalha-se o caso de divisão por um número inteiro e depois por uma fração (cf. Quadro 7). Outros contextos, relacionados à culinária, alimentação e dinheiro, também foram identificados (PHILIPP, 2000; PIEL; GREEN, 1994; SHARP; ADAMS, 2002).

\begin{tabular}{l|l|l}
$\begin{array}{l}\text { Dividir uma corda de } \frac{4}{7} \text { de pés uniformemente } \\
\text { em dois segmentos mais curtos e informe } \\
\text { quanto mede cada segmento. }\end{array}$ & $\begin{array}{l}\text { Se um caminhão viaja 20 milhas em } \frac{2}{5} \text { de hora, quantas milhas } \\
\text { ele viajará em l hora? } \\
\text { O problema é resolvido usando duas } \\
\text { abordagens: }\end{array}$ \\
$\begin{array}{l}\text { 1. Pense o procedimento de dividir } \frac{4}{7} \text { de um } \\
\text { problema é } 20 \div \frac{2}{5} \text {. }\end{array}$ \\
$\begin{array}{l}\text { pé em dois segmentos como sendo dividir } \\
\text { quatro segmentos de } \frac{1}{7} \text { de pé em dois grupos } \\
\text { equivalentes. Cada grupo deverá conter dois } \\
\text { segmentos de } \frac{1}{7} \text { de pé, ou } \frac{2}{7} \text { de pé. Assim, } \frac{4}{7} \div\end{array}$
\end{tabular}


$2=\frac{4 \div 2}{7}=\frac{2}{7}$ de um pé.

2. Pense o procedimento de dividir $\frac{4}{7}$ de um pé igualmente e dois segmentos como encontrar o comprimento de cada segmento. Este problema é equivalente a achar $1 / 2$ de $\frac{4}{7}$ de um pé, ou seja, $\frac{4}{7} \div 2=\frac{4}{7} \times \frac{1}{2}=\frac{2}{7}$ de um pé.

A solução ao problema leva para a primeira regra: a fração dividida por um número inteiro (exceto 0) é igual a fração multiplicada pelo inverso do número inteiro.
Viaja 20 milhas em 2/5 de hora

O primeiro passo é achar quantas milhas o caminhão viaja em $\frac{1}{5}$ de hora. Nós sabemos que ele viaja 20 milhas em dois $\frac{1}{5}$ de horas, então, nós precisamos calcular $20 \div 2$, ou $20 \times \frac{1}{2}$ milha. O segundo passo é achar quantas milhas o caminhão viaja em uma hora. Nós sabemos que uma hora é cinco $\frac{1}{5}$ de horas, então, precisamos calcular $20 \times \frac{1}{2} \times 5$ ou $20 \times \frac{2}{5}$.

Usando este raciocínio, a solução é $20 \div \frac{2}{5}=20 \times \frac{5}{2}=50$ milhas.

A solução ao problema leva a uma segunda regra: um número inteiro dividido por uma fração é igual ao número multiplicado pelo inverso da fração.

Quadro 7 - Uma sistematização da divisão de frações a partir de problemas contextualizados Fonte: Li (2008) traduzido e adaptado pelos autores (2019).

Abordagem com recursos didáticos. Outro viés para ensinar divisão de frações é utilizando materiais manipuláveis (incluindo tiras de papel, jogos, fitas, barbantes, tesouras etc.) e recursos tecnológicos. Bulgar (2003) elaborou problemas relacionados a descobrir quantos laços de diversos comprimentos fracionados podem ser feitos com vários tamanhos de corda, por exemplo, quantos laços de um terço de metro de comprimento podem ser feitos com uma corda de seis metros? Estudantes tiveram acesso a materiais (como fita, barbante, tesoura e fita métrica) e inventaram métodos próprios de resolução. Eles colaboraram, experimentaram, hipotetizaram, testaram hipóteses, construíram conceitos e se orgulharam das realizações. Os resultados obtidos se contrapõem a estudos que detectam dificuldades de alunos em resolver problemas envolvendo frações. O autor alerta que, para desenvolver esta abordagem, é preciso haver tempo suficiente para explorar profundamente as ideias dos alunos e respeitá-las.

Em relação aos recursos tecnológicos, Moreira (2013) utilizou o software Calculadora de Fração para propor atividades aos alunos que os desafiavam a descobrir como o programa obtinha os resultados da divisão de frações. Os estudantes tiveram dificuldade, mas chegaram à generalização do algoritmo de Inverter e Multiplicar. Avaliações escritas antes e depois da intervenção mostram avanço no conhecimento dos estudantes, passando de nenhum para quase todos os alunos resolvendo corretamente divisões de fração.

No que diz respeito a jogos, o trabalho de Flores (2013) apresenta uma atividade que utiliza um quebra-cabeça e uma tabela de frações (Quadro 8). Peréz (2009) descreve outros materiais manipulativos lúdicos (como dominós, triminós de frações e transparências de 
quadrados divididos), suas características e possibilidades de uso. Materiais estes que muitos professores desconhecem (ARAÚJO, 2013).

TAREFA MATEMÁTICA

a) Utilizando a Tabela de Fracões e o quebra cabeça de frações podemos ver que "1/2 é o dobro de $1 / 4$ ". Completa as seguintes frases utilizando os materiais.

$\frac{1}{2}$ contém __ vezes o $\frac{1}{8}$

Se parto pela metade o $\frac{1}{4}$ obtenho está contido 2 vezes em $\frac{1}{3}$

A quarta parte do dobro de $\frac{1}{2}$ é

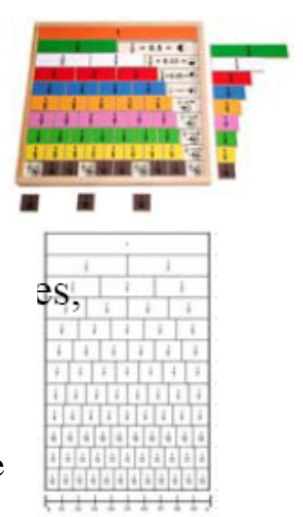

b) “ $1 / 2$ é o dobro de $1 / 4$ ” pode ser expressado simbolicamente das seguintes formas:

$$
\frac{1}{2}=2 \times \frac{1}{4} \quad \frac{1}{2} \div \frac{1}{4}=2 \quad \frac{1}{4} \div \frac{1}{2}=2
$$

Expresse as frases anteriores em forma simbólica (item a), usando operações com frações.

c) Busque novas comparações multiplicativas de frações, utilizando os materiais didáticos e as expresse de forma verba e simbólica.

Quadro 8 - Tarefa matemática escolar para aprendizagem da divisão de frações Fonte: Flores (2013, tradução nossa).

Implementar as propostas anteriores pode ser desafiador para muitos professores, pois exige lidar com as estratégias intuitivas e particulares que estudantes criam quando resolvem problemas, muitas das quais, serão novas para os docentes (SHARP; ADAMS, 2002). A capacidade de dar explicações instrucionais que respondam às dúvidas dos alunos está diretamente correlacionada com sua habilidade de identificar erros dos alunos e suas prováveis fontes (BAYOUD, 2011; LO; MCCRORY, 2010). Por exemplo, pode parecer difícil para crianças aceitarem que o resultado de uma divisão seja maior que o dividendo, como em $\frac{2}{3} \div \frac{1}{3}=2$ e $\frac{2}{3} \div \frac{1}{6}=4$. É importante considerar o nível escolar ou de desenvolvimento conceitual em que o aluno está para decidir como justificar adequadamente certos aspectos matemáticos.

Em Tirosh (2000), a maioria dos licenciandos optou por usar a intepretação medida (característica-chave 2) nos dois exemplos anteriores para explicar a alunos porque dobrar o denominador do divisor implica em dobrar o quociente da divisão de frações, acreditando que isto seja mais acessível aos alunos do que justificativas formais. Este e outros aspectos da aprendizagem de estudantes são discutidos a seguir.

\subsection{Aspectos da aprendizagem de estudantes}

Nesta seção apresentamos resultados sobre (i) erros comuns de estudantes e fontes prováveis, (ii) dificuldades e estratégias de solução que eles implementam quando resolvem problemas contextualizados, (iii) características de apreensão do conteúdo e (iv) metas de aprendizagem que identificamos em estudos do corpus que investigaram a divisão de frações 
e sua aprendizagem (AKSU, 1997; ASHLOCK, 2006; BERTONI, 2009; BULGAR, 2003; CONTRERAS, 2012; FLORES, 2013; LI, 2008; LOPES, 2008; MOREIRA, 2013; NAISER; WRIGHT; CAPRARO, 2003; PURITZ, 2005; SHARP; ADAMS, 2002), bem como, o conhecimento docente sobre esta temática (BAYOUD, 2011; ÖZEL, 2013; TIROSH, 2000).

Alguns trabalhos utilizaram três categorias de erros comuns de estudantes (ÖZEL, 2013; TIROSH, 2000). Na primeira, estão os de base algorítmica que ocorrem quando se subverte um ou mais passos de um algoritmo memorizado, por exemplo, inverter o dividendo e multiplicar pelo divisor, desconhecimento ou confusão dos passos necessários. Na segunda estão os equívocos de base intuitiva que resultam de generalizações equivocadas de propriedades das operações com inteiros para as com frações, por exemplo, pensar que o divisor deve ser um número inteiro, ou que deve ser menor que o dividendo e que o quociente deve ser menor que o dividendo. Na terceira estão os de base formal relacionados a conhecimentos incorretos sobre frações e propriedades da divisão de frações, por exemplo, acreditar que tal operação é comutativa.

Os licenciandos investigados em Tirosh (2000) souberam identificar sete tipos de erros de estudantes no início da formação e três no final (cf. quadro a seguir). Ao final do curso, a minoria se referiu a erros formais (por exemplo, não saber que o inverso de 4 é $\frac{1}{4}$ pode levar alunos a escrever -4), a maioria usou duas categorias de erro (algorítmico e intuitivo) e alguns, todas as três. Em Özel (2013) os professores consideraram equivocadamente que o algoritmo DND e ID eram erros de estudantes.

\begin{tabular}{|c|c|}
\hline Erros para $\frac{\mathbf{1}}{4} \div \mathbf{4}=?$ & $\frac{1 \div 4}{4 \div 4}$ ou $\frac{1}{4} \times 4$ ou $\frac{1}{4 \div 4}$ ou $\frac{4}{1} \times 4$ \\
\hline Erros para $\frac{\mathbf{1}}{\mathbf{4}} \div \frac{\mathbf{3}}{\mathbf{5}}=?$ & $\frac{1}{4} \times \frac{3}{5}$ ou $\frac{3}{4 \div 5}$ \\
\hline Erros para $4 \div \frac{\mathbf{1}}{\mathbf{4}}=?$ & $4 \div 4$ ou $\frac{1}{4} \times 4$ \\
\hline Erros para $320 \div \frac{\mathbf{1}}{\mathbf{3}}=?$ & $320 \div 3$ \\
\hline
\end{tabular}
Fonte: Tirosh (2000).

Outros dois erros são identificados na literatura (ASHLOCK, 2006; FLORES, 2013). O primeiro consiste em aplicar o algoritmo Dividir Numeradores e Denominadores entre si, porém, ignorar o resto em ambas divisões $\left(\frac{5}{7}: \frac{2}{2}=\frac{2}{3}\right)$. Uma provável fonte deste erro é o aluno não saber o conceito de fração e considerá-la como dois números (sempre inteiros) separados por um traço. O segundo erro é quando o estudante sabe inverter e multiplicar, mas inverte a primeira fração ao invés da segunda $\left(\frac{2}{3}: \frac{3}{8}=\frac{3}{2} \cdot \frac{3}{8}=\frac{9}{16}\right)$. Trata-se de um equívoco algorítmico, demonstrando falta de compreensão conceitual da divisão de frações e do uso de habilidades numéricas e de estimativa, considerando que o resultado daquela divisão é maior do que 1 , 
pois $\frac{2}{3}$ (que é mais da metade de alguma coisa) contém pelo menos uma vez o $\frac{3}{8}$ (que é menos da metade da mesma coisa).

Um estudo utilizando estes erros mostrou que os professores foram melhores em identificar padrões de erros de estudantes do que os licenciandos, bem como, em propor atividades para a superação dos mesmos (BAYOUD, 2011). Isto reforça a defesa de que professores devem se familiarizar desde a formação inicial com processos cognitivos comuns, por vezes equivocados, de estudantes ao dividir frações e os efeitos de se usar tais processos (LO; MCCRORY, 2010; TIROSH, 2000). Devem conhecer também atividades instrucionais para ajudar os estudantes a superarem tais equívocos, a exemplo das sugeridas por Ashlock (2006), incluindo descobrir um padrão em expressões corretas e estimar respostas usando comparação em uma grandeza (característica-chave 2) por meio de tiras de papel, materiais manipuláveis ou linha numérica. Tais conhecimentos interferem na qualidade das explicações instrucionais que os docentes vão dar, conforme vimos na seção anterior.

Em relação a erros, quando estudantes lidam com problemas contextualizados, é comum a divisão por $\frac{1}{2}$ ser interpretada como dividir por 2 , encontrar a metade de (PURITZ, 2005). Os estudantes podem ter dificuldade em identificar situações de divisão de frações em problemas que envolvem comparação ou área (FLORES, 2013) e fazê-lo melhor quando se tratam de problemas de distribuição de frações por um inteiro (CONTRERAS, 2012). Dificuldades também foram vistas num grupo de 155 estudantes de $6^{\circ}$ ano, no qual houve menor desempenho na solução de problemas do que em expressões numéricas de divisão de frações (AKSU, 1997).

Pode ocorrer também de estudantes não identificarem o resultado da divisão com relação ao problema (FLORES, 2013). Por exemplo, para repartir $1 \mathrm{~kg}$ de arroz em pacotes de $\frac{2}{5}$ de $\mathrm{kg}$ pode-se pensar que 1 contém $\frac{2}{5}+\frac{2}{5}+\frac{1}{5}$, e portanto a resposta (errada) seria $2+\frac{1}{5}$ pacotes, desconsiderando que este resto $\left(\frac{1}{5}\right)$ deve ter como referência o "tamanho" do pacote. A resposta correta seria $2+\frac{1}{2}$ pacotes, pois o $\frac{1}{5} \mathrm{~kg}$ só encherá meio pacote de $\frac{2}{5} \mathrm{~kg}$.

Além de erros e dificuldades, investigações sobre as estratégias de solução de problemas elaboradas por estudantes mostraram características deste processo. No estudo de Sharp e Adams (2002) foi comum alunos usarem figuras, símbolos e palavras para resolver as situações e comunicar as soluções. Neste processo, ficou clara a mobilização de conhecimentos prévios (sobre adição e subtração de frações e uma definição de divisão de inteiros) e a construção de conhecimento sobre divisão de frações, na medida em que alguns 
estudantes desenvolveram procedimentos simbólicos formais e outros procedimentos pictóricos, dentre eles o algoritmo de denominadores comum. $\mathrm{O}$ algoritmo inverter e multiplicar não foi inventado pelos sujeitos. A descoberta de regras também foi identificada em outros estudos (MOREIRA, 2013; NAISER; WRIGHT; CAPRARO, 2003), assim como o uso de conhecimentos prévios sobre números inteiros e frações (BULGAR, 2003), além do uso da ideia de comparação em uma grandeza (característica-chave 2).

Em termos de características de apreensão do conteúdo, resultados de um estudo mostram que números mistos podem atuar como distratores e dificultam o reconhecimento da divisão por estudantes (CONTRERAS, 2012). Os números $\frac{1}{2}, \frac{1}{4}$ e $\frac{1}{8}$ são identificados como mais confortáveis para alunos no início da aprendizagem de divisão de frações e $\frac{1}{6}, \frac{1}{10}$ e $\frac{1}{12} \mathrm{o}$ são posteriormente (SHARP; ADAMS, 2002). Terços, quintos, metades e quartos são consideradas frações compreensíveis para alunos desde o $5^{\circ}$ ano (BERTONI, 2009). Também foi sugerido que alunos entre o $4^{\circ}$ e o $6^{\circ}$ anos não "dispõem de ferramental algébrico" (LOPES, 2008, p. 17) suficiente para dar sentido à regra geral de divisão de frações.

O estabelecimento de conexões entre objetos concretos e expressões numéricas abstratas é uma tarefa difícil para crianças, por isso a visualização de uma representação figura geométrica (em geral retangular ou circular), desenho (como de pizza ou chocolate), objeto manipulativo ou símbolo - por si só não é suficiente para que o aluno desenvolva a compreensão do uso da divisão de frações para resolver problemas (LO; MCCRORY, 2010). Como vimos na seção anterior, é necessário explicações que incluam referência explícita a significados e relações entre as quantidades, figuras ou expressões envolvidas na situação.

Em termos de metas de aprendizagem, percebemos que a capacidade de resolver problemas de divisão de frações é um aspecto importante e, por vezes, principal. Encontramos dois trabalhos que apresentaram explicitamente as normas de aprendizagem da divisão de frações presentes em documentos oficiais de seu país. Uma delas indica a perspectiva chinesa, na qual os estudantes devem construir conhecimento do significado e das regras computacionais da divisão de frações, porém, devem desenvolver habilidade de resolver problemas envolvendo esta operação mais do que a proficiência em usar algoritmos (LI, 2008). A resolução de problemas também está presente na outra perspectiva, a espanhola, a qual estabelece que os alunos devem ser capazes de "traduzir uma expressão simbólica de divisão mediante um problema de comparação" (FLORES, 2013, p. 12, tradução nossa). Nenhum estudo apresentou a perspectiva brasileira, disponível em documentos oficiais. 


\section{Considerações finais}

Neste trabalho identificamos as principais contribuições de estudos sobre conhecimento docente relativo ao ensino e a aprendizagem da divisão de frações e analisar como tais resultados contribuem para responder a pergunta: que conjunto de conhecimentos um professor precisa para ensinar e fazer aprender divisão de frações? A resposta encontrada no corpus investigado é por nós compreendida como uma proposta de conhecimentos especializados que professores podem (ou devem) ter para ensinar divisão de frações (CARRILLO et al., 2014; MORIEL JUNIOR, 2014) e consiste no conjunto de conhecimentos involucrados em cinco blocos: 1. Definições e notações; 2. Algoritmos, suas justificações e outros procedimentos; 3. Interpretações e problemas associados; 4. Explicações instrucionais, propostas de ensino e recursos didáticos; 5 . Aspectos da aprendizagem de estudantes.

Em termos de conhecimentos matemáticos identificamos aqueles relativos a: conceitos prévios (ligados a operações com naturais e inteiros, significados de frações e a ideia de comparação e proporção), definições formais da divisão de frações do ponto de vista avançado (utilizando classe de equivalência ou operador), fundamentação desta operação da Educação Básica a partir da Matemática do Ensino superior, três tipos de notações, sete algoritmos e suas justificações (tanto aritmética, quanto algébrica), três regras mnemônicas para realizar a divisão de frações, dois tipos de procedimentos alternativos de resolução baseados na manipulação de diagramas pictóricos com formas geométricas ou imagens de objetos (resolução com desenhos) ou de materiais, diferentes tipos de significados, interpretações e problemas associados que modelam a divisão de frações (com seis características-chave diferentes), bem como, a associação entre interpretações, problemas e sistematização do algoritmo associado.

Em termos de conhecimento didático, identificamos o seguinte: diferença entre explicações instrucionais do tipo enunciar a matemática e ensinar para compreensão conceitual e procedimental da divisão de frações, elementos a contemplar na preparação e execução de uma boa explicação instrucional do conteúdo, seis abordagens para o ensino baseado na sequenciação de problemas matemáticos ou contextualizados (word problems) com ou sem uso de materiais manipulativos e tecnológicos, diferentes tipos e exemplos de erros comuns de estudantes no conteúdo e fontes prováveis, processos cognitivos comuns, dificuldades e estratégias de solução discentes frente a problemas contextualizados, características facilitadoras e complicadoras de apreensão do conteúdo e metas de aprendizagem para o conteúdo no sistema escolar. 
Ainda que sintetizemos os conhecimentos em domínios separados (matemático e didático), é justamente a visão integradora dos mesmos o nosso resultado mais importante, sendo expressa pelas conexões de uns com outros, tanto dentro de cada bloco ou domínio, quanto entre eles. A gama de estudos investigados mostra que conhecer como se resolve uma divisão de frações algoritmicamente não é o mesmo que conhecer como criar uma situaçãoproblema que corresponda a uma divisão de frações, atividade esta que se mostrou desafiadora para muitos (futuros) professores. Tampouco, é suficiente para ajudar os estudantes da Educação Básica a desenvolverem por completo a compreensão deste conceito.

A capacidade de dar boas explicações instrucionais está relacionada à habilidade de lidar com os erros dos estudantes e suas possíveis fontes. As propostas identificadas mostram que existem abordagens para ensinar divisão de frações sem que a regra seja colocada em primeiro lugar. O que elas têm em comum é a ideia de que o estudante seja envolvido em atividades que lhe possibilite primeiro uma compreensão conceitual e, posteriormente, o leve a deduzir a regra. Planejar e conduzir propostas de ensino baseadas em problemas ou recursos manipulativos/tecnológicos envolve, dentre outros, o conhecimento das metas de aprendizagem do conteúdo para determinado nível do sistema escolar, dos significados e interpretações da divisão de frações, dos tipos de problemas associados, de como sistematizar um algoritmo de divisão de fração a partir das soluções (muitas vezes intuitivas, particulares e baseadas em desenhos) dos alunos frente a um problema com determinada característicachave associada ao conteúdo.

Os resultados desta meta-análise estão situados no âmbito do corpus construído a partir de seis bancos de dados, embora existam outras fontes possíveis, em virtude da limitação dos recursos humanos, financeiros e temporais disponíveis pelos autores para a consecução do mesmo. Por isso, faz-se necessária a produção de mais estudos de síntese (neste tema ou em outros) para aprofundar a compreensão, a abrangência, o impacto e a divulgação dos resultados de pesquisa na Educação Matemática.

Nosso estudo oferece uma contribuição significativa e é útil a licenciandos, professores, formadores e pesquisadores, na medida em que podem extrair daqui dimensões para análise e reflexão sobre o ensino e a aprendizagem de divisão de frações, com foco na superação das limitações de conhecimento profissional. Consequentemente, se pode caminhar no sentido da melhoria da prática e da formação docente, seja a sua própria ou de outrem.

Como desdobramentos desta pesquisa, sinalizamos a necessidade de empreender esforços em novas investigações que utilizem os resultados desta meta-análise para desenvolver atividades formativas baseadas em situações de prática, bem como, analisar seu 
alcance na construção de conhecimentos profissionais na formação inicial e continuada. Neste sentido, há uma indissociabilidade entre a teoria e a prática, de modo que a formação didática considera o domínio matemático e a formação matemática leva em consideração o domínio didático (MORIEL JUNIOR; CYRINO, 2009).

Um exemplo disto seria analisar o potencial formativo do uso do Quadro 4 (com características-chave de problemas, exemplos e análise dimensional) na preparação de profissionais ao planejar e executar o ensino de divisão de frações por meio da abordagem de resolução de problemas. Também acreditamos ser um caminho pertinente ampliar a quantidade de estudos sobre abordagens de ensino (descritas aqui ou não) para buscar informações tão esclarecedoras quanto possível sobre as condições e situações em que o uso de uma tende a ser mais adequado do que de outra. Estes são aspectos importantes para orientar as escolhas didáticas, pois refletir sobre os diferentes modos que os professores desenvolvem os conceitos ajuda a discernir como/se estão funcionando e esta informação pode ser usada para aprimorar tanto as aulas, quanto a formação docente.

Em síntese, este trabalho trata da caracterização de um panorama de conhecimentos que são especializados (CARRILLO et al., 2013; MORIEL JUNIOR, 2014) a partir de resultados de investigações calcados na prática profissional do ensino e da aprendizagem de divisão de frações. Panoramas deste tipo fornecem elementos que podem auxiliar (futuros) professores a ampliar a compreensão sobre o próprio conhecimento, refinando-o e aprofundando-o e, ainda, serem fontes úteis para formadores de professores desenvolverem atividades visando a construção do conhecimento especializado docente. Também podem ser úteis na elaboração de designs inovadores de cursos de formação (a exemplo da licenciatura em Matemática, da Faculdade SESI de Educação, SP), nos quais se espera a presença daquilo que há de mais avançado na Educação Matemática.

Acreditamos que esta pesquisa ajuda a reforçar a tese de que o professor de Matemática precisa ter uma gama de conhecimentos especializados para ensinar Matemática, cuja construção demanda uma formação especializada com formadores que possuam tais conhecimentos especializados. Assumir esta posição leva a implicações mais amplas sobre ações e políticas relacionadas não só aos tempos e componentes curriculares de formação, mas também em relação à própria valorização da profissão e à criação/manutenção de condições adequadas de preparação e trabalho. 


\section{Agradecimentos}

Este trabalho contou com fomento da FAPEMAT (Edital Universal 42/2016), apoio do IFMT (Edital 49/2017/PROPES e Resolução 10/2015/CONSUP) e da Coordenação de Aperfeiçoamento de Pessoal de Nível Superior - Brasil (CAPES).

\section{Referências}

AKSU, M. Student performance in dealing with fractions. The Journal of Educational Research, London, v. 90, n. 6, p. 375-380, 1997.

ARAÚJO, W. A. D. O uso do FRAC-SOMA 235 no processo de ensino e aprendizagem de frações para o ensino fundamental. In: Encontro Nacional de Educação Matemática, 11., 2013, Curitiba. Anais... Curitiba, 2013. p. 1-10. Disponível em: http://sbem.bruc.com.br/XIENEM/pdf/72_1718_ID.pdf . Acesso em: 14 out. 2014.

ASHLOCK, R. B. Error patterns in computation: Using error patterns to improve instruction. Upper Saddle River: Pearson Education, 2006.

BALL, D. L. The Subject Matter Preparation of Prospective Mathematics Teachers: Challenging the Myths. Michigan. Research Reports NCRTE-RR-88-3, 1988, p. 1-43. Disponível em: http://files.eric.ed.gov/fulltext/ED301468.pdf . Acesso em: 14 out. 2014.

. Prospective elementary and secondary teachers' understanding of division. Journal for research in mathematics education, Delaware, v. 21, n. 2, p. 132-144, 1990.

BALL, D. L.; HILL, H. C.; BASS, H. Knowing mathematics for teaching: Who knows mathematics well enough to teach third grade, and how can we decide? American Educator, Washington, v. 29, n. 3, p. 14-46, 2005. Disponível em: http://www.aft.org/pdfs/americaneducator/fall2005/BallF05.pdf . Acesso em: 14 out. 2014.

BALL, D. L.; THAMES, M. H.; PHELPS, G. Content Knowledge for Teaching: What Makes It Special? Journal of teacher education, San Francisco, v. 59, n. 5, p. 389-407, 2008.

BARBOSA, E. P. Os Por Quês Matemáticos dos Alunos na Formação dos Professores. In: Conferência Interamericana de Educação Matemática - CIAEM, 13., 2011, Recife. Anais... Recife, 2011. p. 1-12. Disponível em: http://www.cimm.ucr.ac.cr/ocs/files/conferences/1/schedConfs/1/ papers/611/public/611-9763-1-PB.pdf. Acesso em: 14 out. 2014.

BAYOUD, J. M. A comparison of pre-service and experienced elementary teachers' pedagogical content knowledge (PCK) of common error patterns in fractions. 2011. 180 p. Thesis (Master of Arts). Departament of Education, American University of Beirut, Beirut.

BERTONI, N. E. A construção do conhecimento sobre número fracionário. Bolema: Boletim de Educação Matemática, Rio Claro, v. 21, n. 31, p. 209-237, 2008.

Educação e linguagem matemática: Frações e números fracionários. Curitiba: PEDEad, 2009. 95 p. Disponível em: < http://www.sbembrasil.org.br/files/fracoes.pdf >. Acesso em: 14 out. 2014.

BICUDO, M. A. V. Meta-análise: seu significado para a pesquisa qualitativa. REVEMAT: Revista eletrônica de educação matemática, Florianópolis, v. 9, p. 7-20, 2014. 
BRASIL. Sistema Nacional de Avaliação da Educação Básica - SAEB. Brasília: MEC, 2001.

. Sistema Nacional de Avaliação da Educação Básica - SAEB. Brasília: MEC, 2003.

BULGAR, S. Using Research to Inform Practice: Children Make Sense of Division of Fractions.

International Group for the Psychology of Mathematics Education, Honolulu, v. 2, p. 157-164, 2003. Disponível em: http://files.eric.ed.gov/fulltext/ED500921.pdf . Acesso em: 14 out. 2014.

CARRILLO, J.; CLIMENT, N.; CONTRERAS, L. C.; MONTES, M. Á.; ESCUDERO, D.; MEDRANO, E. F. Un marco teórico para el Conocimiento especializado del Profesor de Matemáticas. Huelva: Universidad de Huelva Publicaciones, 2014. 93 p.

CARRILLO, J.; CLIMENT, N.; CONTRERAS, L. C.; MUÑOZ-CATALÁN, M. C. Determining Specialised Knowledge For Mathematics Teaching. In: UBUZ, B.; et al (Ed.). VIII Congress of the European Society for Research in Mathematics Education (CERME 8). Antalya, Turkey: Middle East Technical University, Ankara, 2013. p. 2985-2994.

CHARALAMBOUS, C. Y.; HILL, H. C.; BALL, D. L. Prospective teachers' learning to provide instructional explanations: how does it look and what might it take? Journal of Mathematics Teacher Education, Utrecht, v. 14, n. 6, p. 441-463, 2011.

CHEN, C.-H.; CHIU, C.-H.; LIN, C.-P.; WU, S.-T.; HUNG, Y.-C. Presenting Solution Strategies of Fraction Multiplication and Division on Mathematics Instructional Websites. World Journal on Educational Technology, Nicosia, v. 5, n. 3, p. 431-444, 2013. Disponível em: http://worldeducation-center.org/index.php/wjet/article/view/1068 . Acesso em: 14 out. 2014.

CHINNAPPAN, M.; DESPLAT, B. Contextualisation of Fractions: Teachers' Pedagogical and Mathematical Content Knowledge for Teaching. Journal of Science and Mathematics Education in Southeast Asia, Gelugor, v. 35, n. 1, p. 43-59, 2012.

CONTRERAS, M. La división de fracciones: un algoritmo misterioso. In: Jornades d'Educación Matemática de la Comunitat Valenciana, 6., 2004, Valencia. Anais... Valencia: Societat "AlKhwarizmi", 2004. p. 1-13 Disponível em: http://www.mauriciocontreras.es/UN ALGORITMO MISTERIOSO.pdf . Acesso em: 14 out. 2014.

. Problemas multiplicativos relacionados con la división de fracciones: un estudio sobre su enseñanza y aprendizaje. 2012. 374f. Tese (Doutorado em Didática da Matemática) Departament de Didàctica de les Matematiques, Universidad de Valencia, Valencia, 2012.

CONTRERAS, M.; ALFONSO, B. G. Estudio de algunos problemas multiplicativos en los que interviene la división de fracciones. In: Seminario Investigación en Educación Matemática, 9., 2005, Cordoba. Anais... Cordoba: SEIEM, 2005. p. 1-9.

FÁVERO, M. H.; PINA NEVES, R. D. S. A divisão e os racionais: revisão bibliográfica e análise. Zetetiké, Campinas, v. 20, n. 1, p. 39-78, 2012. Disponível em: http://www.fae.unicamp.br/zetetike/viewarticle.php?id=553 . Acesso em: 14 out. 2014.

FIORENTINI, D.; LORENZATO, S. Investigação em Educação Matemática: percursos teóricos e metodológicos. Campinas: Autores Associados, 2006. 224 p .

FLORES, P. El algoritmo de la división de fracciones. Epsilon, Sevilla, v. 25, n. 70, p. 27-40, 2008.

FLORES, P. ¿Por qué multiplicar en cruz? Formación inicial de profesores de Primaria, en el área de Matemáticas. In: VII Congreso Iberomericano de Educación Matemática, 7., 2013, Montevideo.

Anais... Montevideo: CIBEM, 2013. p. 1-17. 
GARCÍA, A. I. M. Conocimiento profesional de un grupo de profesores sobre la división de fracciones. 2013. 72 p. Dissertação (Mestrado Didática da Matemática) - Universidad de Granada, Granada, 2013.

GREEN, M.; PIEL, J. A.; FLOWERS, C. Reversing Education Majors' Arithmetic Misconceptions with Short-Term Instruction Using Manipulatives. Journal of Educational Research, Philadelphia, v. 101, n. 4, p. 234-242, 2008. Disponível em:

http://www.tandfonline.com/doi/pdf/10.3200/JOER.101.4.234-242. Acesso em: 14 out. 2014.

GUERRA, R. B.; SILVA, F. H. S. D. As Operações com Frações e o Princípio da Contagem. Bolema: Boletim de Educação Matemática, Rio Claro, v. 21, n. 31, p. 41-54, 2008. Disponível em: http://www.redalyc.org/pdf/2912/291221883004.pdf. Acesso em: 14 out. 2014.

HIRATSUKA, P. I. Fazendo uma divisão de frações significativa. Revista do Professor de Matemática, Rio de Janeiro, v. 30, p. 23-25, 1996.

INEP. Banco de dados do SAEB de 1995 a 2005. Brasília: MEC, 2014a. Disponível em: http://download.inep.gov.br/educacao_basica/prova_brasil_saeb/resultados/matematicauf(urb_s_fed)95-05-8a.xls. Acesso em: 16 ago 2014.

Banco de dados do SAEB de 2011. Brasília: MEC, 2014b. Disponível em:

http://download.inep.gov.br/educacao_basica/prova_brasil_saeb/resultados/2012/Saeb_2011_primeiro s_resultados_site_Inep.pdf. Acesso em: 16 ago 2014.

ISIKSAL, M.; CAKIROGLU, E. Pre-service teachers' representations of division of fractions. In: PANTAZI, D. P.; PHILIPPOU, G. (Ed.). Proceedings of the Fifth Congress of the European Society for Research in Mathematics Education. Cyprus: European Research in Mathematics Education V, 2007. p. 1916-1924.

KAMII, C.; WARRINGTON, M. A. Division with Fractions: A Piagetian, Constructivist Approach. Hiroshima Journal of Mathematics Education, Hiroshima, v. 3, p. 53-62, 1995.

KILPATRICK, J.; SWAFFORD, J.; FINDELL, B. Adding it up: Helping children learn mathematics. Washington: National Academies Press, 2001. Disponível em: http://www.sjsd.k12.mo.us/cms/lib3/MO01001773/Centricity/Domain/872/Adding it Up.pdf. Acesso em: 14 out. 2014.

KRIBS-ZALETA, C. Invented strategies for division of fractions. In: ALATORRE, S. et al (Ed.). Proceedings of the 28th annual meeting of the North American Chapter of the International Group for the Psychology of Mathematics Education. Meridia: Universidad Pedagógica Nacional, v. 2, 2006. p. 371-376.

LI, Y. What Do Students Need to Learn about Division of Fractions? Mathematics Teaching in the Middle School, Reston, v. 13, n. 9, p. 546-552, 2008. Disponível em: http://www.jstor.org/stable/41182613. Acesso em: 14 out. 2014.

LI, Y.; CHEN, X.; AN, S. Conceptualizing and organizing content for teaching and learning in selected Chinese, Japanese and US mathematics textbooks: the case of fraction division. ZDM, Berlin, v. 41, n. 6, p. 809-826, 2009. Disponível em: http://dx.doi.org/10.1007/s11858-009-0177-5. Acesso em: 14 out. 2014.

LI, Y.; CHEN, X.; KULM, G. Mathematics teachers' practices and thinking in lesson plan development: a case of teaching fraction division. ZDM, Berlin, v. 41, n. 6, p. 717-731, 2009.Disponível em: http://dx.doi.org/10.1007/s11858-009-0174-8. Acesso em: 14 out. 2014. 
LI, Y.; KULM, G. Knowledge and confidence of pre-service mathematics teachers: the case of fraction division. ZDM, Berlin, v. 40, n. 5, p. 833-843, 2008. Disponível em:

http://dx.doi.org/10.1007/s11858-008-0148-2. Acesso em: 14 out. 2014.

LI, Y.; SMITH, D. Prospective middle school teachers'knowledge in mathematics and pedagogy for teaching-the case of fraction division. In: WOO, J.-H. et al (Ed.). Proceedings of the 31st conference of the international group for the Psychology of Mathematics Education. Seoul, Korea: ERIC, v. 3, 2007. p. 185-192.

LIMA, E. L. Divisão de números racionais escritos na forma de frações. Revista do Professor de Matemática, Rio de Janeiro, v. 3, p. 40-42, 1983.

LIMA, T. C. S.; MIOTO, R. C. T. Procedimentos metodológicos na construção do conhecimento científico: a pesquisa bibliográfica. Revista Katálysis, Florianópolis, v. 10, p. 37-45, 2007. Disponível em: http://www.scielo.br/pdf/rk/v10nspe/a0410spe.pdf. Acesso em: 14 out. 2014.

LIN, C.-Y.; BECKER, J.; BYUN, M.-R.; YANG, D.-C.; HUANG, T.-W. Preservice Teachers' Conceptual and Procedural Knowledge of Fraction Operations: A Comparative Study of the United States and Taiwan. School Science and Mathematics, West Lafayette, v. 113, n. 1, p. 41-51, 2013. Disponível em: http://dx.doi.org/10.1111/j.1949-8594.2012.00173.x. Acesso em: 14 out. 2014.

LO, J.-J.; LUO, F. Prospective elementary teachers' knowledge of fraction division. Journal of Mathematics Teacher Education, Utrecht, v. 15, n. 6, p. 481-500, 2012. Disponível em: http://dx.doi.org/10.1007/s10857-012-9221-4. Acesso em: 14 out. 2014.

LO, J.-J.; MCCRORY, R. Teaching Teachers through Justifying Activities. Teaching Children Mathematics, Reston, v. 17, n. 3, p. 149-155, 2010. Disponível em: http://eric.ed.gov/?q=fraction+division+knowledge\&pg=2\&id=EJ902166. Acesso em: 14 out. 2014.

LOPES, A. J. O que nossos alunos podem estar deixando de aprender sobre frações, quando tentamos lhes ensinar frações. Bolema: Boletim de Educação Matemática, Rio Claro, v. 21, n. 31, p. 1-22, 2008. Disponível em: http://www.periodicos.rc.biblioteca.unesp.br/index.php/bolema/issue/view/756. Acesso em: 14 out. 2014.

LUBINSKI, C. A.; FOX, T.; THOMASON, R. Learning to Make Sense of Division of Fractions: One K-8 Preservice Teacher's Perspective. School Science and Mathematics, West Lafayette, v. 98, n. 5, p. 247-259, 1998. Disponível em: http://dx.doi.org/10.1111/j.1949-8594.1998.tb17298.x. Acesso em: 14 out. 2014.

LUO, F.; LO, J.-J.; LEU, Y.-C. Fundamental Fraction Knowledge of Preservice Elementary Teachers: A Cross-National Study in the United States and Taiwan. School Science and Mathematics, West Lafayette, v. 111, n. 4, p. 164-177, 2011. Disponível em: http://dx.doi.org/10.1111/j.19498594.2011.00074.x. Acesso em: 14 out. 2014.

MA, L. Knowing and teaching elementary mathematics: Teachers' understanding of fundamental mathematics in China and the United States. New York: Taylor \& Francis, 1999. 198 p.

MADEIRO, P. C. Divisão de fração por fração. Revista do Professor de Matemática, Rio de Janeiro, v. 30, p. 22, 1996.

MOREIRA, I. M. B. O ensino das operações com frações envolvendo calculadora. In: VII Congreso Iberomericano de Educación Matemática, 7., 2013, Montevideo. Anais... Montevideo, 2013. p. 29963005. Disponível em: http://www.cibem7.semur.edu.uy/7/actas/pdfs/1231.pdf. Acesso em: 14 out. 2014. 
MOREIRA, P. C.; DAVID, M. M. M. S. O conhecimento matemático do professor: formação e prática docente na escola básica. Revista Brasileira de Educação, Rio de Janeiro, v. 11, n. 28, p. 5062, 2005. Disponível em: http://www.scielo.br/pdf/rbedu/n28/a05n28.pdf. Acesso em: 14 out. 2014.

MOREIRA, P. C.; FERREIRA, M. C. C. A Teoria dos Subconstrutos e o Número Racional como Operador: das estruturas algébricas às cognitivas. Bolema: Boletim de Educação Matemática, Rio Claro, v. 21, n. 31, p. 103-127, 2008. Disponível em:

http://www.redalyc.org/pdf/2912/291221883007.pdf. Acesso em: 14 out. 2014.

MORIEL JUNIOR, J. G. Conhecimento especializado para ensinar divisão de frações. 2014. 162 p. Tese (Doutorado em Educação em Ciências e Matemática) - PPGECEM/REAMEC, Universidade Federal de Mato Grosso, Cuiabá, 2014.

MORIEL JUNIOR, J. G.; CYRINO, M. C. C. T. Propostas de articulação entre teoria e prática em cursos de licenciatura em matemática. Educação Matemática Pesquisa, São Paulo, v. 11, n. 3, p. 535-557, 2009. Disponível em: http://revistas.pucsp.br/index.php/emp/article/viewArticle/2831. Acesso em: 14 out. 2014.

NAISER, E. A.; WRIGHT, W. E.; CAPRARO, R. M. Teaching fractions: Strategies used for teaching fractions to middle grades students. Journal of research in childhood education, New York, v. 18, n. 3, p. 193-198, 2003.

NEWTON, K. J. An Extensive Analysis of Preservice Elementary Teachers' Knowledge of Fractions. American Educational Research Journal, Michigan, v. 45, n. 4, p. 1080-1110, dec. 2008. Disponível em: http://aer.sagepub.com/content/45/4/1080. Acesso em: 14 out. 2014.

NILLAS, L. Division of fractions: Preservice teachers' understanding and use of problem solving strategies. The Mathematics Educator, Athens, v. 7, n. 2, p. 96-113, 2003.

OLANOFF, D. E. Mathematical Knowledge for Teaching Teachers: The Case of Multiplication and Division of Fractions. 2011. 236f. Thesis (Doctor of Mathematics Education). Syracuse University, New York, 2011.

ÖZEL, S. An Analysis of In-service Teachers' Pedagogical Content Knowledge of Division of Fractions. Anthropologist, New York, v. 16, n. 1-2, p. 1-5, 2013.

PAYNE, J. N. Review of research on fractions. In: LESH, R. (Ed.). Number and measurement. University of Georgia: Athens, v. 1, 1976. p. 145-188.

PERÉZ, B. F. Materiales para la enseñanza de las fracciones. Revista Digital Innovación y Experiencias Educativas, Granada, n. 24, p. 1-8, 2009. Disponível em: http://www.csicsif.es/andalucia/modules/mod_ense/revista/pdf/Numero_24/BLANCA_FERNANADEZ_1.pdf. Acesso em: 14 out. 2014.

PHILIPP, R. A. Unpacking a conceptual lesson: The case of dividing fractions. San Diego, 2000. Research Reports of Project Grant G00002211, p. 1-15. Disponível em: http://www.sci.sdsu.edu/CRMSE/IMAP/pubs/Unpacking.pdf. Acesso em: 14 out. 2014.

PIEL, J. A.; GREEN, M. De-Mystifying Division of Fractions: The Convergence of Quantitative and Referential Meaning. Focus on learning problems in mathematics, Framingham, v. 16, n. 1, p. 4450, 1994. Disponível em: http://eric.ed.gov/?q=fraction+division+knowledge\&pg=3\&id=EJ485504 . Acesso em: 14 out. 2014. 
PINILLA, M. I. F. Fractions: conceptual and didactic aspects. Acta Didactica Universitatis Comenianae, Bratislava, n. 7, p. 81-115, 2007. Disponível em:

http://www.ddm.fmph.uniba.sk/ADUC/files/Issue7/05Pinilla.pdf. Acesso em: 14 out. 2014.

PURITZ, C. Dividing by Small Numbers - and Why Not by 0? Mathematics in School, Leicester, v. 34, n. 5, p. 2-4, 2005. Disponível em: http://www.jstor.org/stable/30215826. Acesso em: 10 Maio 2012 .

REDMOND, A. Prospective Elementary Teachers' Division of Fractions Understanding: A Mixed Methods Study. 2009. 178 p. Thesis (Doctor of Philosophy) - University of Phoenix, Stillwater, 2009.

RIZVI, N. F.; LAWSON, M. J. Prospective teachers' knowledge: Concept of division. International Education Journal, Adelaide, v. 8, n. 2, p. 377-392, 2007. Disponível em: http://ehlt.flinders.edu.au/education/iej/articles/v8n2/Rizvi/paper.pdf. Acesso em: 14 out. 2014.

RPM. Revista do Professor de Matemática. São Paulo: Sociedade Brasileira de Matemática (USP), 1982-2009. 70 vol. CD ROM. 2011.

RULE, A. C.; HALLAGAN, J. E. Preservice Elementary Teachers Use Drawings and Make Sets of Materials to Explain Multiplication and Division by Fractions. In: Annual Preparing Mathematicians to Educate Teachers, 2006, New York. Proceedings... New York, 2006. p. 1-22. Disponível em: http://files.eric.ed.gov/fulltext/ED494956.pdf. Acesso em: 14 out. 2014.

SÁ, I. P. D. Voltando aos "porquês" nas aulas de matemática. Rio de Janeiro: UERJ, p. 1-6, 2015. Disponível em: http://magiadamatematica.com/uerj/licenciatura/09-voltando1.pdf. Acesso em: 01 nov. 2014.

SALINAS, T. M. Beyond the Right Answer: Exploring How Preservice Elementary Teachers Evaluate Student-Generated Algorithms. The Mathematics Educator, Athens, v. 19, n. 1, p. 27-34, 2009.

SALVADOR, A. D. Métodos e técnicas de pesquisa bibliográfica. Porto Alegre: Sulina, 1986.

SANTOS, R. S. D.; SANTOS, M. C. D.; CAMPOS, T. M. M. Estratégias utilizadas pelos alunos da educação básica na resolução de questões sobre números racionais na prova do saepe/sistema de avaliação educacional de Pernambuco. In: ENCONTRO NACIONAL DE EDUCAÇÃO MATEMÁTICA - ENEM, 11., 2013, Curitiba. Anais... Curitiba, 2013. p. 1-15. Disponível em: http://sbem.esquiro.kinghost.net/anais/XIENEM/pdf/1500_1265_ID.pdf. Acesso em: 14 out. 2014.

SHARON, V. V.; SWARTHOUT, M. B. Evaluating instruction for developing conceptual understanding of fraction division. In: MATNEY, G. T.; CHE, S. M. (Ed.). Proceedings of the 41th Annual Meeting of the Research Council on Mathematics Learning. San Antonio: RCML, 2014. p. $97-104$.

SHARP, J.; ADAMS, B. Children's constructions of knowledge for fraction division after solving realistic problems. The Journal of Educational Research, New York, v. 95, n. 6, p. 333-347, 2002.

SHULMAN, L. S. Those who understand: Knowledge growth in teaching. Educational researcher, New York, v. 15, n. 2, p. 4-14, 1986.

SILVA, M. J. F.; ALMOULOUD, S. A. As Operações com Números Racionais e seus Significados a partir da Concepção Parte-todo. Bolema: Boletim de Educação Matemática, Rio Claro, v. 21, n. 31, p. 55-78, 2008. Disponível em: http://www.leoakio.com/wa_files/2105-8989-2-PB.pdf. Acesso em: 14 out. 2014. 
SILVEIRA, M. R. A. D.; SILVA, P. V. A compreensão de regras matemáticas na formação docente: uma pesquisa sob o ponto de vista da linguagem. Education Policy Analysis Archives, Tempe, v. 21, n. 27, p. 1-24, 2013. Disponível em: http://epaa.asu.edu/ojs/article/view/1161. Acesso em: 14 out. 2014.

SINICROPE, R.; MICK, H. W.; KOLB, J. R. Interpretations of fraction division. In: LITWILLER, B. (Ed.). Making sense of fractions, ratios, and proportions. Reston: National Council of Teachers of Mathematics (NCTM), 2002. p. 153-161.

TCHOSHANOV, M. A. Relationship between teacher knowledge of concepts and connections, teaching practice, and student achievement in middle grades mathematics. Educational Studies in Mathematics, Utrecht, v. 76, n. 2, p. 141-164, 2011.

TIROSH, D. Enhancing prospective teachers' knowledge of children's conceptions: The case of division of fractions. Journal for Research in Mathematics Education, Delaware, p. 5-25, 2000.

Submetido em 03 de Abril de 2018. Aprovado em 26 de Maio de 2019. 